\title{
99\% beta factor and directional coupling of quantum dots to fast light in photonic crystal waveguides determined by spectral imaging
}

\author{
L. Scarpelli, ${ }^{1}$ B. Lang, ${ }^{2,}{ }^{*}$ F. Masia,,${ }^{1}$ D. M. Beggs, ${ }^{1}$ E. A. Muljarov, ${ }^{1}$ A. B. Young, ${ }^{2}$ R. Oulton, ${ }^{2}$ M. Kamp, ${ }^{3}$ S. Höfling, ${ }^{3}$ \\ C. Schneider, ${ }^{3}$ and W. Langbein ${ }^{1}$ \\ ${ }^{1}$ School of Physics and Astronomy, Cardiff University, The Parade, Cardiff CF24 3AA, United Kingdom \\ ${ }^{2}$ Quantum Engineering Technology Labs, School of Physics and Department of Electrical \& Electronic Engineering, University of Bristol, \\ Nanoscience and Quantum Information Building, Tyndall Avenue, Bristol, BS8 1FD, United Kingdom \\ ${ }^{3}$ Technische Physik, Physikalisches Institut and Wilhelm Conrad Röntgen Center for Complex Material Systems, Universität Würzburg, \\ Am Hubland, 97474 Würzburg, Germany
}

(Received 7 April 2019; published 30 July 2019)

\begin{abstract}
Spontaneous emission from excitonic transitions in InAs/GaAs quantum dots embedded in photonic crystal waveguides at $5 \mathrm{~K}$ into nonguided and guided modes is determined by direct spectral imaging. This enables measurement of the absolute coupling efficiency into the guided modes, the beta factor, directly, without assumptions on decay rates used previously. Notably, we found beta factors above $90 \%$ over a wide spectral range of $40 \mathrm{meV}$ in the fast light regime, reaching a maximum of $(99 \pm 1) \%$. We measure the directional emission of the circularly polarized transitions in a magnetic field into counterpropagating guided modes, to deduce the mode circularity at the quantum dot sites. We find that points of high directionality, up to $97 \%$, correlate with a reduced beta factor, consistent with their positions away from the mode field antinode. By comparison with calibrated finite-difference time-domain simulations, we use the emission energy, mode circularity, and beta factor to estimate the quantum dot position inside the photonic crystal waveguide unit cell.
\end{abstract}

DOI: 10.1103/PhysRevB.100.035311

\section{INTRODUCTION}

Quantum dots (QDs) embedded in photonic crystal waveguides (PCWGs) are a promising system to implement quantum technologies. Due to the broadband coupling [1,2], the system can be used for high-efficiency on-chip single-photon sources. Furthermore, the strong lateral confinement of light results in a significant longitudinal component of the electromagnetic mode field, which allows for local circular polarization, and therefore selective coupling of circularly polarized dipoles into a single mode [3-8]. Recent experiments have shown QD spin-photon path conversion and photon pathdependent QD spin initialization using this mechanism $[9,10]$, which is robust against disorder [11]. In conjunction with the recently demonstrated spin-controlled photon switching [12] and superradiant emission from two coupled QDs in a PCWG [13], these results show the potential of such a system for the implementation of scalable quantum technologies on chip $[14,15]$. A fundamental requisite of quantum technologies based on QD on PCWGs is that the spontaneous emission (SE) of the emitter couples exclusively to the designed channels of the system, which are typically waveguide (WG) modes, and not to other background channels creating losses. For a given WG mode, the probability of a QD exciton (QDE) to emit into the mode is called the beta factor, defined as

$$
\beta=\frac{\Gamma_{\mathrm{wg}}}{\Gamma_{\mathrm{wg}}+\Gamma_{\mathrm{ng}}+\Gamma_{\mathrm{nr}}},
$$

${ }^{*}$ Current address: School of Physics and Astronomy, University of Nottingham, UK. where $\Gamma_{\mathrm{wg}}$ and $\Gamma_{\mathrm{ng}}$ are the decay rates to the selected WG mode and other, typically nonguided, modes, respectively, and $\Gamma_{\mathrm{nr}}$ is the nonradiative decay rate. In previous works, the beta factor was estimated using lifetime measurements [16-21]. The challenge in these measurements is to determine all three decay rates. A common approach assumed $\Gamma_{\mathrm{wg}}$ to be given by the difference between the decay rate of a QDE coupled to the WG mode $\Gamma_{\mathrm{c}}$ and the decay rate of similar QDEs not coupled to the WG, $\Gamma_{\mathrm{nc}}$, so that $\Gamma_{\mathrm{wg}}=\Gamma_{\mathrm{c}}-\Gamma_{\mathrm{nc}}$, and $\Gamma_{\mathrm{ng}}+\Gamma_{\mathrm{nr}}=\Gamma_{\mathrm{nc}}$. The accuracy of this analysis depends on the accuracy of the underlying assumption that all QDEs have the same decay rate into nonguided modes, and the same nonradiative decay. The nonradiative decay $\Gamma_{\mathrm{nr}}$ is dependent on QD charging and local defects and is thus determined by properties beyond the photonic environment. Assuming no influence of local defects or tunneling, it is negligible for neutral excitons in InAs/GaAs QDs. Even in charged excitons, where Auger processes provide a nonradiative decay, this rate in the order of $1 \mu \mathrm{s}^{-1}$ [22] is three orders of magnitude below typical radiative decay rates of $1 \mathrm{~ns}^{-1}$ [23]. The radiative decay into other modes $\Gamma_{\text {ng }}$, however, is likely to be significantly modified by the local dielectric environment of the QDs in PCWG structures, as recent calculations [24] have highlighted. Therefore, the analysis reported in previous works is expected to exhibit significant systematic errors in the determined beta factor, as recently pointed out [25].

In this work we use direct spectral imaging to determine the emitted power, avoiding assumptions on decay rates altogether. The SE from QDs embedded in the PCWG along the WG is imaged onto the input slit of an imaging spectrometer, and is measured spatially and spectrally resolved. In this 

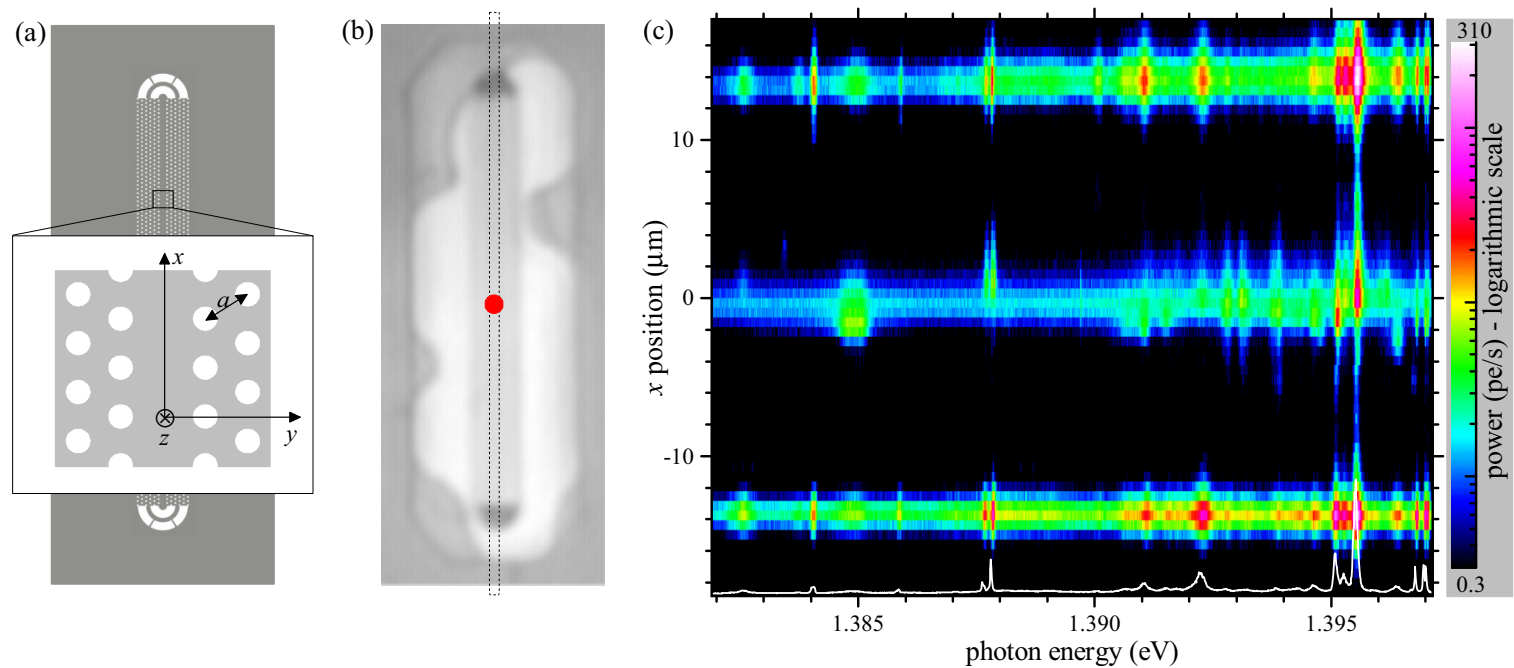

FIG. 1. Sample and spectral imaging. (a) Structure of the investigated PCWG. The zoom shows the lattice structure and the reference system used throughout. (b) White light reflection image of $\mathrm{WG}_{\mathrm{A}}$. The red circle represents the spot of the excitation laser and the black dashed line indicates the region corresponding to the input slit of the spectrometer. (c) Spectral image from the full CCD camera display, in units of photoelectrons per time and per pixel. The bottom and top regions are the QD emission into the WG mode and coupled to free space by the bottom and top couplers, respectively. The central region is the direct free-space emission from the QDs. White line: spectrum emitted from the bottom coupler.

way, the SE guided by the WG and coupled at the ends of the WG by grating couplers into free space is measured together with the SE emerging from the QD directly into free space. The beta factor is determined using the relative emission powers, after correcting for the propagation losses and relative efficiencies of the couplers. Using an external magnetic field in Faraday (out-of-plane) direction, the QDE states are split into two spectrally resolvable transitions with opposite circular polarization. Depending on the QD location within the unit cell of the PCWG, the two transitions couple differently to WG modes of opposite propagation direction. Using the spectrally resolved emission from the two couplers, the emission into the two counterpointing WG modes is measured. Using the powers of the two transitions emerging from the two couplers, we quantify the directionality $[6,9]$ of the emission, and deduce the WG mode directionality $D$ at the QD site. The statistical distribution of the determined beta factor versus $D$ over a large ensemble of QDs, which are expected to randomly sample the in-plane area of the PCWG, shows that $\beta$ above $90 \%$ are mostly found for directionalities below $80 \%$, and vice versa. The experimental results are analyzed using detailed electromagnetic simulations of the PCWG structure. Specifically, we calculate $\beta$ and $D$ versus position inside the PCWG, and comparing with experiments, we estimate the QD position within the PCWG unit cell on a 10-nm length scale. This position is expected to affect the exciton dephasing, both via the interaction with surface states and through a modification of the local phonon density of states [26].

\section{SAMPLE AND METHODS}

The investigated sample is a GaAs photonic crystal membrane of $125-\mathrm{nm}$ nominal thickness, with a single layer of
InAs/GaAs QDs at the center. The QD area density is about $10^{9} \mathrm{~cm}^{-2}$, corresponding to an average distance of about 300 $\mathrm{nm}$, or about one QD per unit cell of the PCWG. The QDs are $n$ doped using a Si delta-doping layer with a density of about $10^{10} \mathrm{~cm}^{-2}, 10 \mathrm{~nm}$ below the QDs. Given the inhomogeneous size and spatial distribution of the QDs, this leads to a distribution of QD electron charging. Multiply charged QDs provide broader SE multiplets due to final-state damping and spin splitting. The emission lines analyzed were sharp lines which did not show a resolvable fine-structure splitting. Since typical values of fine-structure splittings for these QDs are in the few $10-\mu \mathrm{eV}$ range [27], and considering the doping, we attribute these lines to negatively charged exciton transitions. Even though the charging is relevant for applications using the electron spin [7,12], it is not important for the experimental results shown in this work. A fine-structure splitting much smaller than the magnetic-field-induced Zeeman splitting of around $50 \mu \mathrm{eV}$, however, is important for the purity of the circular polarization of the transitions in a magnetic field [28].

The PCWG is created by a line of missing holes in the periodic hexagonal pattern of round air holes of separation $a$, giving rise to guided modes within the two-dimensional (2D) photonic band gap for modes with dominating in-plane electric fields [29]. An illustration of the investigated PCWGs is shown in Fig. 1(a). The PCWGs investigated here are $100 a$ long, with $a=260 \mathrm{~nm}$, and have six rows of holes on each side. The PCWGs are terminated by Bragg reflector couplers, which couple light propagating in the PCWG to free space [30]. Two PCWGs (called $\mathrm{WG}_{\mathrm{A}}$ and $\mathrm{WG}_{\mathrm{B}}$ ) have been analyzed, which differ by their hole radius to period ratio $r / a$, being 0.24 and 0.26 , respectively.

The sample is mounted in a low-vibration closed-cycle cryostat (Montana Cryostation) on a XYZ piezostage (Attocube) with a spatial resolution around $0.1 \mu \mathrm{m}$, allowing 
focusing and lateral alignment. For the measurements shown in this work, the sample temperature was $5 \mathrm{~K}$. A microscope objective (MO) with a numerical aperture (NA) of 0.85 is mounted inside the cryostat on the cold shield, having a temperature of about $30 \mathrm{~K}$. This avoids sample heating by thermal radiation, which can be an issue, specifically with membrane structures due to their reduced thermal contact to the substrate, when using the more common geometry with a room-temperature window close to the sample and an external MO. A permanent magnet can be mounted below the sample to provide a magnetic field of $B_{z}=0.45 \mathrm{~T}$, where $z$ labels the direction normal to the sample plane, as indicated in Fig. 1(a).

The QD SE was excited by a laser at a wavelength of $\lambda=633 \mathrm{~nm}$ focused onto the sample to a sub- $\mu \mathrm{m}$ spot by the MO (see also Appendix F1). The SE is filtered with a color filter (Schott RG680) transmitting wavelengths above $680 \mathrm{~nm}$. The SE from the QDs can be visualized by two imaging cameras, one for the near field (NF), imaging the real space at the sample, and one for the far field (FF), imaging the reciprocal space at the sample. For spectral imaging, the real space is imaged onto the input slit of an imaging spectrometer with a focal length of $1.9 \mathrm{~m}$, a $1200-1 / \mathrm{mm}$ holographic grating of $(120 \times 140) \mathrm{mm}^{2}$ size, $900-\mathrm{nm}$ blaze wavelength, and detected by a CCD (Roper Pixis) of $1340 \times 100$ square pixels of $20-\mu \mathrm{m}$ size. For all the measurements performed in this work, the input slit aperture was $20 \mu \mathrm{m}$, corresponding to $639 \mathrm{~nm}$ at the sample plane. The corresponding spectral resolution [full width at half maximum (FWHM)] is $8 \mu \mathrm{eV}$ at $880 \mathrm{~nm}$ $(1.41 \mathrm{eV})$. A white light Köhler illumination is integrated with the main photoluminescence (PL) setup, to simultaneously visualize the PCWG sample and the PL emission from the QDs. The origin of the $x$ axis along the WG is chosen at the center of the WG.

A reflection image of the sample is shown in Fig. 1(b). The red spot indicates the excitation laser at a specific position along the WG. The excitation at a photon energy of $1.96 \mathrm{eV}$ (633-nm wavelength) creates electron-hole pairs in the GaAs membrane, which subsequently relax by phonon emission toward the GaAs band gap around $1.52 \mathrm{eV}$, before being captured into the highly strained InGaAs wetting layer, where they further relax to the wetting layer band gap around $1.42 \mathrm{eV}$, and finally into the QDs, which emit in the energy range between 1.37 and $1.41 \mathrm{eV}$. The direct imaging allows the measurement of the diffusion length in the planar region of the sample, which was found to be about $3-4 \mu \mathrm{m}$ on the membrane, as shown in Appendix F1. The described carrier relaxation process is complex, with the formation of excitons in GaAs and the wetting layer also playing a role. Once captured into a QD, the carriers relax to the ground state within tens of picoseconds, from which they radiatively recombine, emitting a photon into the local photonic mode structure, consisting of the WG modes, confining light, and nonguided modes, rapidly escaping to free space on both sides of the slab. The emission is imaged onto the input slit of the spectrometer, indicated as black dashed line in Fig. 1(b). Importantly, the WG has been aligned along the slit, in order to collect and image the emission from the whole WG and the couplers. The SE spectrum from the QDs is therefore detected spatially resolved along the $\mathrm{WG}$, as exemplified in
Fig. 1(c), covering an energy range of about $15 \mathrm{meV}$ for a given spectrometer center position. Exciting at $x=0 \mu \mathrm{m}$, we observe most of the free-space emission close to the excitation, while signals around $x= \pm 13 \mu \mathrm{m}$ arise from the WG couplers, representing the QD SE into the WG modes. The spectrum from the bottom WG coupler is indicated as a white line, integrated over $y \in[-14.3,-11.4] \mu \mathrm{m}$. The center signal, close to the excitation position, is attributed to QD SE into the nonguided modes, excited by the carrier relaxation and diffusion processes described before. However, we also observed QD free-space emission from positions far away from the excitation spot, which we attribute to indirect excitation, where the wetting layer emission, which is coupled into the WG mode, is absorbed by QD excited states (see Appendix B).

\section{PHOTONIC BAND STRUCTURE}

Determining the photonic band structure of the investigated sample is crucial for a quantitative comparison with numerical simulations. In the literature, near-field scanning optical spectroscopy [31] and interferometric techniques [32] have been used to determine the guided mode dispersion in PCWGs. In other cases, the simulation parameters were adjusted to approximately reproduce the measured transmission window [33]. Alternatively, Fabry-Pérot fringes were used to calculate the group index of the guided mode [21].

Here, we use Fourier imaging [34,35] to directly measure the band dispersion within the light cone [36]. We use pulsed laser excitation, with center energies $E_{\mathrm{L}}$ corresponding to band wave vectors within the light cone, coupled to the WG via the bottom coupler. The laser polarization is set orthogonal to the WG direction, in order to select the fundamental mode (see Appendix G3). To suppress the reflection of the excitation laser, we use a rectangular aperture in an intermediate image plane, corresponding to $(8.7,19.0)-\mu \mathrm{m}$ size at the PCWG. We measure both the NF and the FF of the emission along the WG as a function of energy, from which we can determine the propagation losses and the wave vector of the corresponding Bloch mode. An example of a NF measurement for $E_{\mathrm{L}}=$ $1.4565 \mathrm{eV}$ is shown in Fig. 2(b), with the corresponding FF measurement shown in Fig. 2(c). The NF profile along the WG exhibits significant fluctuations, reflecting the coherent nature of the emission. After background subtraction, it can be fit with an exponential decay, as shown in the right panel of Fig. 2(b), from which we obtain the WG loss $\alpha$, as further detailed in Appendix F. The resulting $\alpha$ are given in the inset of Fig. 2(a) as function of $E_{\mathrm{L}}$, showing an increase with $E_{\mathrm{L}}$, which is attributed to both the radiative losses of the WG mode in the light cone and the absorption by the wetting layer, as discussed in more detail in Appendix D.

Turning to the FF measurements, we note that the accessible range of the in-plane wave vector $\mathbf{k}$ is limited by the NA of the MO to $|\mathbf{k}|<k_{0} \mathrm{NA}$, with the free-space wave vector $k_{0}=2 \pi / \lambda$. The resulting cutoff is visible in Fig. 2(c) and from this we can calibrate the $k$ space of our measurements (see Appendix F 3). The measured FF pattern shows a stripe at $k_{x}$ around $-5 \mu \mathrm{m}^{-1}$, elongated in the $k_{y}$ direction, which is the WG radiation loss. The WG mode wave vector is given by $k_{x}$, and the large extension in $k_{y}$ is due to the small 


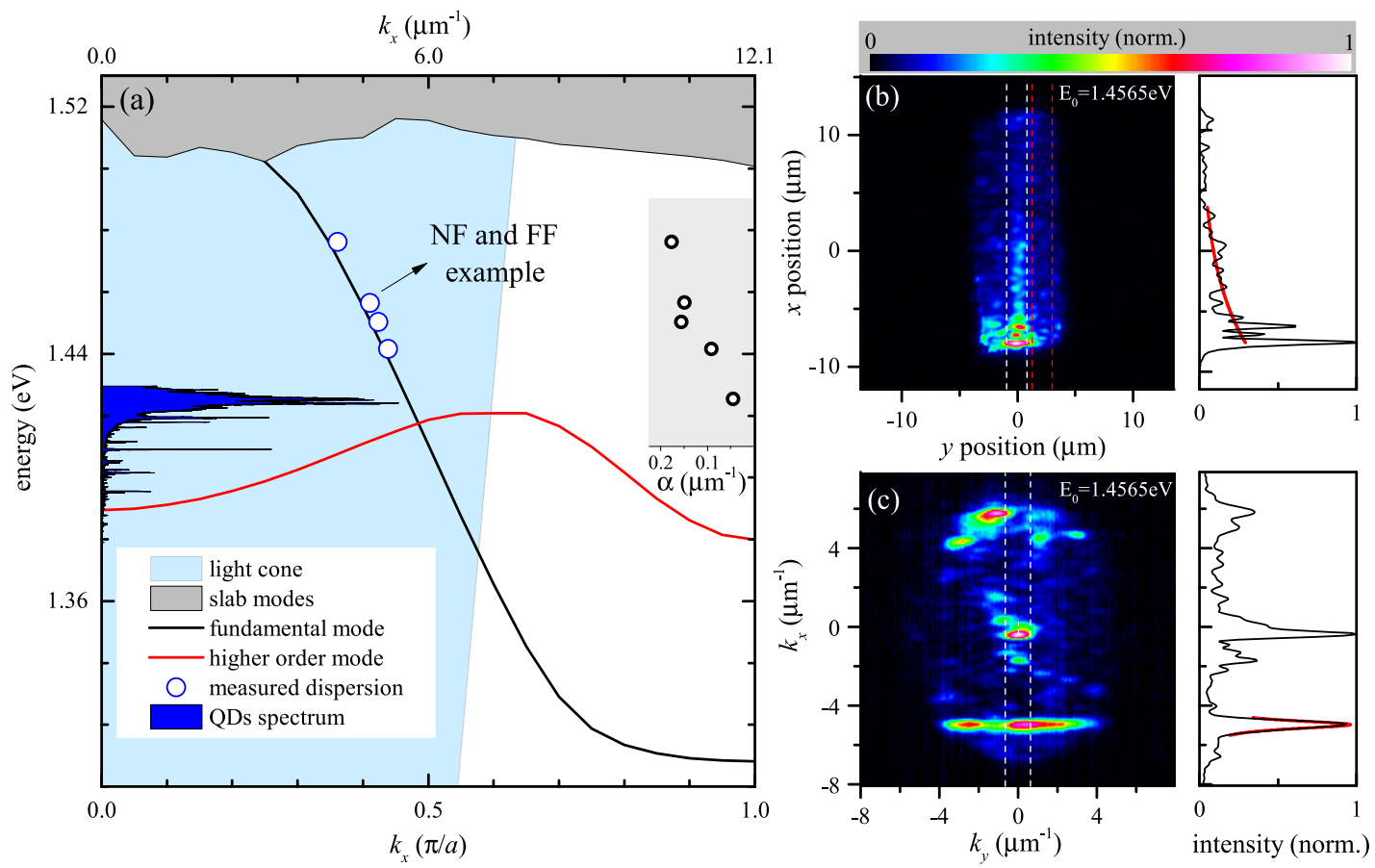

FIG. 2. Photonic band structure and propagation imaging. (a) Simulated photonic band structure of $\mathrm{WG}_{\mathrm{A}}$; dark-blue shaded area: QD emission spectrum; blue circles: measured dispersion from FF imaging; inset: WG loss $\alpha$ obtained from NF imaging. (b) NF image, on color scale as given, and the NF profile (right) obtained as the difference between a cut along the WG and a reference side cut (averages between the white dashed lines and red dashed lines in the image, respectively). Red line: exponential fit. (c) FF image, and FF profile (right) obtained from a cut highlighted by white dashed lines in the image. Red line: Lorentzian fit.

extension of the WG mode in $y$. Knowing that the excited WG mode is propagating in positive $x$ direction, it is interesting to note that it exhibits a negative wave vector $k_{x}$, clear evidence of the negative group velocity of the WG mode, so that the phase velocity, given by $k_{x}$, is opposite to the group velocity, which is along the propagation direction.

The finite width in $k_{x}$ is due to the exponential decay of the field along the $x$ direction, the finite size of the imaged region, and the finite bandwidth of the excitation laser, as discussed in Appendix F 2. We determine the propagation wave vector by fitting the FF profile with a Lorentzian, as shown in the right panel of Fig. 2(c). The FF profile is obtained by averaging over $k_{y} \in[-0.6,0.6] \mu \mathrm{m}^{-1}$, indicated by the white dashed lines. The resulting $\left|k_{x}\left(E_{\mathrm{L}}\right)\right|$ are shown in Fig. 2(a), with the blue circles, noting that the reflection symmetry of the WG allows to use the absolute value.

We now use this measurement of the WG mode wave vectors to fine tune the sample parameters used in simulations. The membrane thickness and hole diameters have been measured by scanning electron microscopy (SEM), and the photonic crystal (PC) period has a negligible fabrication error. We note that the refractive index of GaAs has previously been used as adjustable parameter, taking low-temperature values of $\sqrt{12}$ [1], 3.5 [19], and 3.45 [21]. However, the GaAs refractive index is well known [37] and somewhat larger than these values. We therefore use in our simulations the known refractive index at low temperatures, including its dispersion. The QD layer embedded in the slab corresponds to less than $1 \%$ of its thickness and is made of a similar material as GaAs, so that we neglect its effect on the refractive index.
It is known that fabrication by selective etching, and oxidation of GaAs over time [38,39], can remove a surface layer of GaAs. We therefore use an effective thickness $d$ of a removed surface layer as a parameter, to match the simulations to the measurements, as detailed in Appendix G 1. The calculated band structure for $d=8 \mathrm{~nm}$ is shown in Fig. 2(a), together with a typical free space SE spectrum of the QDs embedded in the PCWG. The measured spectrum consists of sharp lines at energies below $1.41 \mathrm{eV}$, and a broad emission at higher energies, which we attribute to the wetting layer. Sharp lines superimposed to the wetting layer emission are attributed to localized excitons.

\section{RESULTS AND DISCUSSION}

Using the spectral imaging as shown in Fig. 1, we identify the SE of individual QDs from the top and bottom couplers and from the QD position into nonguided modes. The excitation position can be adjusted along the WG to maximize the QD emission. The measurements were performed at a low excitation power to avoid multiexciton emission.

\section{A. Beta factor and directionality}

Examples of the SE detected for three different QDs are shown in Figs. 3(a) $-3(\mathrm{c})$, over a small spectral range covering the splitting of $\Delta_{z} \sim 50 \mu \mathrm{eV}$ created by the magnetic field. As the exciton has spin projection $S_{z}= \pm 1$, the Zeeman splitting between these states is given by $\Delta_{z}=2 \mu_{\mathrm{B}} g_{\mathrm{X}} B_{z}$, with $\mu_{\mathrm{B}}$ the Bohr magneton. Using $B_{z}=0.45 \mathrm{~T}$, we calculate an exciton 

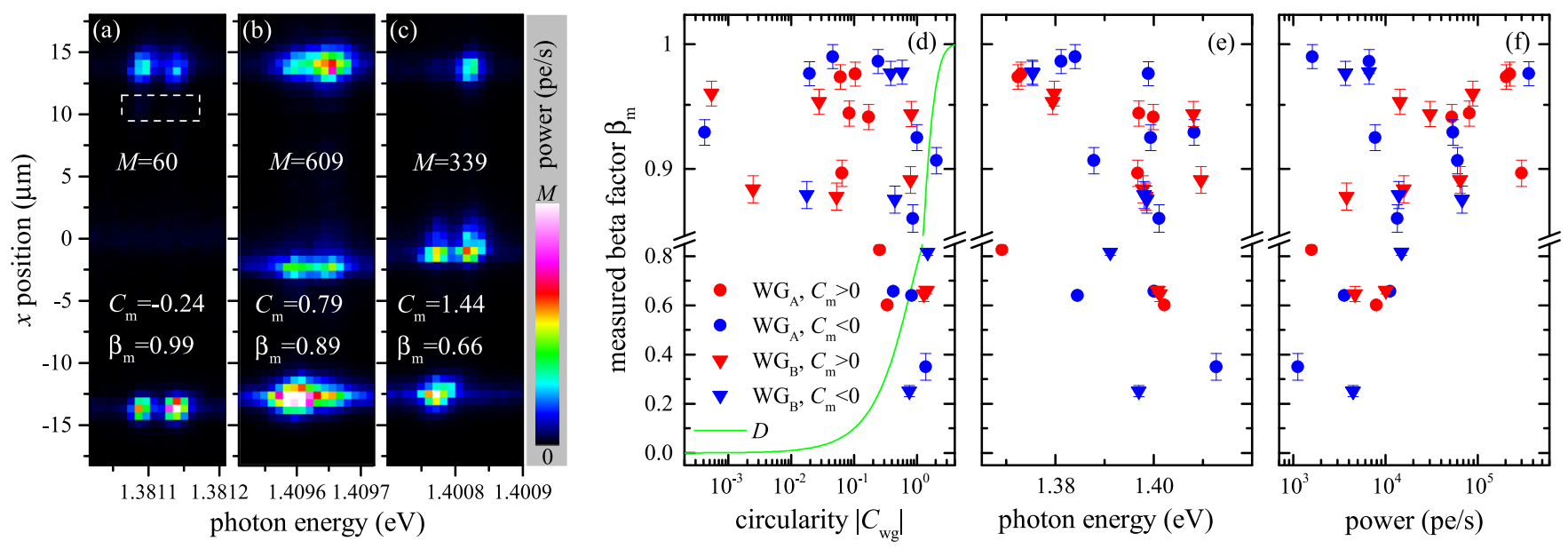

FIG. 3. QD spectral images, beta factor, and directional emission. (a)-(c) Spectral images of the SE from three different QDs located at sites of (a) low, (b) intermediate, and (c) high WG mode circularity. Color scale as shown, from 0 to $M$. The corresponding $C_{\mathrm{m}}$ and $\beta_{\mathrm{m}}$ are given. The weak free-space emission of the QD in (a) is highlighted by the dashed rectangle. (d)-(f) $\beta_{\mathrm{m}}$ as a function of (d) mode circularity $\left|C_{\mathrm{m}}\right|$, (e) QD peak energy, and (f) SE power for local excitation. In (d), the directionality $\left|D_{\mathrm{m}}\right|$ is shown (green line) as a function of $\left|C_{\mathrm{m}}\right|$.

$g$ factor $g_{\mathrm{X}}=0.96$, comparable to the value of 1.2 reported in [9]. The beta factor can be calculated as the ratio between the power emitted from the couplers and the total emitted power as

$$
\beta_{\mathrm{m}}=1-\frac{\sum_{j} P_{\mathrm{fs}}^{j}}{\sum_{j}\left(P_{\mathrm{t}}^{j}+P_{\mathrm{b}}^{j}+P_{\mathrm{fs}}^{j}\right)},
$$

where $P_{\mathrm{b}}, P_{\mathrm{t}}, P_{\mathrm{fs}}$ are the detected SE powers of a single QD from the bottom and top couplers and at the QD site, respectively, corrected for losses and relative coupler efficiencies (see Appendix D), and $j \in\{+,-\}$ labels the helicity of the QD transition. We note that these values are not corrected for the relative collection efficiency of free-space and coupler emission. We discuss the resulting systematic errors in Sec. IV C. The errors of $\beta_{\mathrm{m}}$ given in the following are statistical errors due to measurement noise, as described in Appendix A. Furthermore we determine the QD transition energy and its position along the $\mathrm{WG}, x_{\mathrm{QD}}$, as detailed in Appendix A.

For the QD SE shown in Fig. 3(a), we find $\beta_{\mathrm{m}}=0.99 \pm$ 0.01 . The QD is located close to the top coupler, as highlighted in the figure by the white dashed rectangle. Interestingly, the QD is visible for excitation at $x=0$, which we attribute to reabsorption of the wetting layer emission propagating along the PCWG. We verified that $\beta_{\mathrm{m}}$ is independent of the excitation position (see Appendix B), as expected given that the beta factor is determined by the local photon density of states, which is independent of the QD excitation pathway. Figures 3(b) and 3(c) show the SE of QDs with $\beta_{\mathrm{m}}=0.89 \pm 0.01$ and $\beta_{\mathrm{m}}=0.66 \pm 0.01$, respectively. The directional emission is evident in the asymmetry of the power from top and bottom couplers. The degree of directionality depends on the WG mode circularity $C$ at the QD site, which we calculate here from the measured powers as

$$
C_{\mathrm{m}}=\frac{1}{4} \ln \left(\frac{P_{\mathrm{t}}^{+} P_{\mathrm{b}}^{-}}{P_{\mathrm{t}}^{-} P_{\mathrm{b}}^{+}}\right)
$$

which by using the ratio of power ratios is independent of the efficiencies of the couplers. $C$ is related to the directionality $D$ used in [9] by $D=\tanh (C)$, making it equal for small circularities but avoiding saturation for high circularities [see green line in Fig. 3(d)]. At a circularly polarized point $(C$ point), $C$ diverges, while at a linearly polarized point ( $L$ point), $C$ is zero. Residual reflections from the couplers might limit the maximum value of $C_{\mathrm{m}}$. From the maximum measured $C_{\mathrm{m}}$, we can deduce that these reflections are below $5 \%$ (see Appendix E).

Notably, the $\beta_{\mathrm{m}}$ of the QDs shown in Figs. 3(a)-3(c) decreases with increasing $\left|C_{\mathrm{m}}\right|$. To investigate this further, we determined circularity and beta factor for many QDs, in $\mathrm{WG}_{\mathrm{A}}$ and $\mathrm{WG}_{\mathrm{B}}$, as shown in Fig. 3(d). We find that low $\beta_{\mathrm{m}}$ are more likely at higher circularity. This is consistent with the fact that $C$ points require both transversal and longitudinal fields, and thus do not occur at the mode field antinode. We find positive and negative circularities (red and blue symbols, respectively), randomly distributed for both investigated PCWGs. In the same figure, we show that the corresponding directionality $D$ is above 0.9 for some of the QDs, while in [9] the reported $D$ are below 0.8. Furthermore, we find $\beta_{\mathrm{m}}$ above 0.9 over an emission energy range of about $40 \mathrm{meV}$ [see Fig. 3(e)]. In particular, in $\mathrm{WG}_{\mathrm{A}}$ we find QDs with $\beta_{\mathrm{m}}$ above 0.9 for energies between 1.372 and $1.408 \mathrm{eV}$. We note that in most of this spectral range both fundamental and the higher-order mode are predicted to be present (see Fig. 2). The coupling of a QD to these two modes depends on its energy and its position within the PCWG unit cell, as discussed in Appendix G 6. However, no obvious feature of the presence of the two modes is visible in the dependence of the beta factor on energy [Fig. 3(e)]. Importantly, these results show that efficient coupling occurs even in region of low group index within the light cone. In Fig. 3(f) we plot $\beta_{\mathrm{m}}$ as a function of the total SE power from the QD for excitation at $x_{\mathrm{QD}}$, using for all QDs an excitation power of $0.5 \mu \mathrm{W}$ at the sample. We find $\beta_{\mathrm{m}}$ above 0.90 over three order of magnitudes in SE power. This indicates that the emission power is not dictated by the 


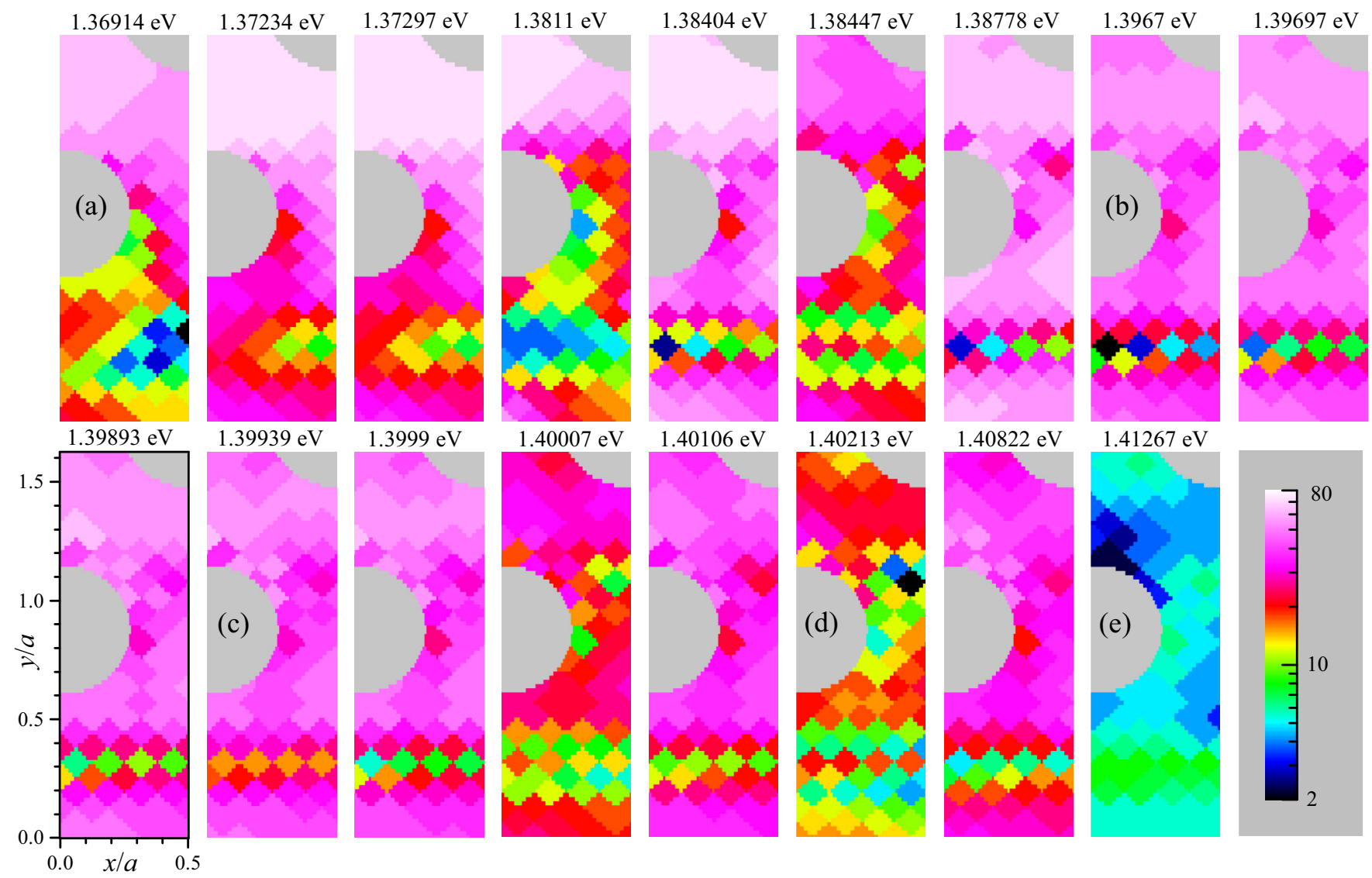

FIG. 4. Determining the QD position in the PCWG unit cell. Residual $\chi(x, y)$ of beta factor and circularity between simulations and experiment according to Eq. (4) for $\mathrm{WG}_{\mathrm{A}}$. Color scale as indicated. Positions $(x, y)$ inside the holes are indicated in gray. The values given are for the closest calculated point, in the center of the diamonds.

beta factor, but is governed mostly by the local carrier capture dynamics, which depends on the disorder landscape in the wetting layer close to the QDs. The circularity and QD energy also do not show a correlation with the coarse QD position along the WG, as shown in Appendix C.

\section{B. Determining the QD position in the PCWG unit cell}

The WG mode circularity at the QD site, together with the QD beta factor and energy, identifies possible positions of the QD inside the PCWG unit cell. We therefore performed finite-difference time-domain (FDTD) simulations for $\mathrm{WG}_{\mathrm{A}}$ with $d=8 \mathrm{~nm}$, extracting the simulated beta factor $\beta_{\mathrm{s}}$ and circularity $C_{\mathrm{s}}$ at positions across the PCWG unit cell, and at the energies of the measured QDEs, as detailed in Appendix G. The simulated positions are organized on a checkerboard grid with a step of $0.125 a=32.5 \mathrm{~nm}$. For a comparison with the measured quantities $\beta_{\mathrm{m}}$ and $C_{\mathrm{m}}$, the simulated beta factor $\beta_{\mathrm{s}}$, and circularity $C_{\mathrm{s}}$ have been corrected to take into account the projections of the QD emission onto the fundamental and higher modes and the corresponding polarization resolved collection efficiencies, which include the couplers, the NA of the $\mathrm{MO}$, the input slit of the spectrometer, and the efficiency of the spectrometer, as detailed in Appendix G, yielding $\tilde{\beta}_{\mathrm{s}}$ and $\tilde{C}_{\mathrm{s}}$. To identify the position of the QD, we compare simulations and experiment taking into account the experimental errors, by evaluating the normalized residual $\chi(x, y)$ for each $\mathrm{QD}$, given by

$$
\chi(x, y)=\sqrt{\left(\chi_{\mathrm{c}}^{2}(x, y)+\chi_{\beta}^{2}(x, y)\right) / 2},
$$

with

$$
\chi_{\mathrm{c}}(x, y)=\frac{C_{\mathrm{m}}-\tilde{C}_{\mathrm{s}}(x, y)}{\Delta C_{\mathrm{m}}}, \quad \chi_{\beta}(x, y)=\frac{\beta_{\mathrm{m}}-\tilde{\beta}_{\mathrm{s}}(x, y)}{\Delta \beta_{\mathrm{m}}} .
$$

In these expressions, $\Delta C_{\mathrm{m}}$ and $\Delta \beta_{\mathrm{m}}$ are the experimental errors (standard deviation) for the measured $C_{\mathrm{m}}$ and $\beta_{\mathrm{m}}$, respectively. $x$ and $y$ are the simulated positions in the unit cell, according to the reference system given in Fig. 1(a). Note that agreement between simulation and experiment within the estimated errors is obtained for values of $\chi$ of the order of unity. In general, the minimum of the residual indicates the most likely position of the QD inside the unit cell. In Fig. 4 the results are shown for QDs in $\mathrm{WG}_{\mathrm{A}}$, ordered with increasing energy. Generally, for most QDs we find positions with residuals below 5. Examples of QDs showing residuals below 2, labeled (a), (b), (d), and (e), enable to extract a well-defined position of the QD, with a precision of a few $10 \mathrm{~nm}$ considering the region over which $\chi$ is increasing by unity (i.e., one standard deviation) from its minimum. These positions are distributed over the unit cell, as expected. One example in (c) shows $\chi>20$ for all simulated positions; possibly, this QD is situated beyond the $y$ range covered in the simulations. 


\section{Systematic errors affecting beta factor measurements}

In the imaging method used in this work, the systematic errors in determining the beta factor are created by different collection efficiencies between free-space emission and WG emission. The collection efficiency of the WG emission is calculated in Appendix G3. It depends on the emission energy, increasing from $10 \%$ to $18 \%$ (see Fig. 19). The collection efficiency of the free-space emission is calculated in Appendix $\mathrm{G} 5$, and varies between $18 \%$ and $33 \%$ depending on the position of the QD in the PCWG unit cell, as given in Fig. 24(b). Note that these values are due to the finite collection angular range of the MO, the finite slit size of the spectrometer, and the polarization dependence of the grating. Other losses in the collection, such as reflections from lenses, or detector quantum efficiencies, which are affecting the two emissions equally, are not influencing $\beta_{\mathrm{m}}$, and are thus not considered here. In the measured beta factor $\beta_{\mathrm{m}}$ shown in Fig. 3 , these collection efficiencies have not been taken into account. They result in a corrected beta factor larger than $\beta_{\mathrm{m}}$. For example, a $\beta_{\mathrm{m}}$ of 0.90 would be corrected to 0.94 using the center values of the efficiency ranges given above. Equivalently, 0.99 would be corrected to 0.995 , and 0.5 to 0.65 , to give a few examples.

Considering instead beta factors determined using decay rates, as reported in [16-21], the systematic error arises from the assumption that the decay rate into nonguided modes $\Gamma_{\mathrm{ng}}$ is equal to the one observed for QDs outside of the photonic crystal slab. We show in Fig. 24(a) that in the structure we have investigated, $\Gamma_{\mathrm{ng}}$ varies over two orders of magnitude depending on the position of the QD in the PCWG unit cell, from $\frac{1}{10}$ to 10 times the bulk rate. This large range leads to large systematic errors of the determined beta factor. For example, a beta factor of 0.90 evaluated using the above assumption corresponds to corrected beta factors ranging from 0.47 to 0.99 . It is therefore very important to calculate the range of $\Gamma_{\text {ng }}$ for the specific structure investigated to determine the systematic errors in decay-rate-based measurements of the beta factor, which was not done in the above works.

\section{CONCLUSIONS}

In conclusion, we have shown that direct spectral imaging allows measurement of the beta factor and the directional emission, without assumptions on radiative decay rates, by using only the relative powers emitted into the WG mode and to free space. We found a maximum beta factor of $(99 \pm 1) \%$ in the fast light regime. Beta factors above $90 \%$ are mainly found for quantum dots located at sites with small WG mode circularity, consistent with the fact that circular points occur away from the field antinodes. Using Fourier imaging to measure the band dispersion of the WG mode within the light cone, we calibrate FDTD simulations, allowing us to locate the QD positions inside the PCWG unit cell with a few 10 $\mathrm{nm}$ precision, from their measured beta factor and circularity. These results are promising for the suitability of the system for photon blockade and more advanced quantum technology, and the methods presented can be used to identify suited QDs. Furthermore, the position determination can be used to determine the precision of $\mathrm{QD}$ site-control techniques, important for the development of useful and scalable devices.
The data presented in this work are available from the Cardiff University data archive [40].

\section{ACKNOWLEDGMENTS}

This work was supported by the EPSRC under Grant No. EP/M020479/1, and partially supported by the GW4 Accelerator "Southwest Quantum Dot Quantum Technologies Grant No. GW4-AF3-004. The simulations were performed in the Advanced Computing Research Centre, University of Bristol. B.L. acknowledges support by an EPSRC studentship Grant No. DTA 1407622. R.O. acknowledges her EPSRC fellowship Grant No. EP/N003381/1. F.M. acknowledges the Ser Cymru II programme (Case ID 80762-CU-148) which is partly funded by Cardiff University and the European Regional Development Fund through the Welsh Government. M.K., S.H., and C.S. acknowledge support by the State of Bavaria. Assistance in sample growth and fabrication by $\mathbf{M}$. Emmerlin, J. Beetz, and S. Maier is gratefully acknowledged.

L.S. performed the measurements, analyzed the data, and drafted the manuscript, with support by F.M., W.L., and B.L. B.L. performed the simulations, with support from D.M.B., E.A.M., L.S., and W.L. A.B.Y., R.O., S.H., and C.S. provided the sample and participated in discussions and manuscript revision. W.L. guided the work.

\section{APPENDIX A: ANALYSIS OF SPECTRAL IMAGES}

Here, we discuss the analysis of spectral imaging data to retrieve the emission powers used in Eqs. (2) and (3). After subtracting the dark background, the data are divided by the integration time and multiplied by the CCD gain of 2 electrons per count, resulting in data as given in Fig. 5(a) in photoelectrons per time and pixel. All data were taken in a magnetic field of $B_{z}=0.45 \mathrm{~T}$, providing a Zeeman splitting into circularly polarized transitions, as visible in Fig. 5(a). In order to determine the total detected power emitted from the QD directly in free space, the emission is integrated along the waveguide over the number of pixels contained in the region of interest. This region is highlighted in Fig. 5(a) with dashed green lines. In order to find the peak positions and areas, we first fit the QD free-space emission using a sum of two Voigt profiles, with equal linewidth parameters, as shown in Fig. 5(c). Using the obtained linewidths and energies, the emission from the couplers is fitted varying the amplitudes only. The areas of the fitted peaks, having units of photoelectrons per time, are taken as the measured powers $P_{i}^{j, \mathrm{~m}}$, with $i \in\{\mathrm{b}, \mathrm{fs}, \mathrm{t}\}$ and $j \in\{+,-\}$. These powers are corrected for WG losses and relative coupler efficiencies, as described in Appendix D, to obtain the powers used to determine $\beta_{\mathrm{m}}$ by Eq. (2). The uncertainties of the peak amplitudes determined by the fit are used to evaluate the corresponding error in $\beta_{\mathrm{m}}$. If the resulting error is below $1 \%$, an error of $1 \%$ is reported, as found from the analysis of independent measurements shown in Fig. 5(d) discussed below.

In order to obtain the $\mathrm{QD}$ position along the $\mathrm{WG}, x_{\mathrm{QD}}$, we first separate the QD signal from the excitation background by averaging over two regions, one including the QD signal and the other one spectrally shifted up by about $100 \mu \mathrm{eV}$ containing the spectrally broad background from 

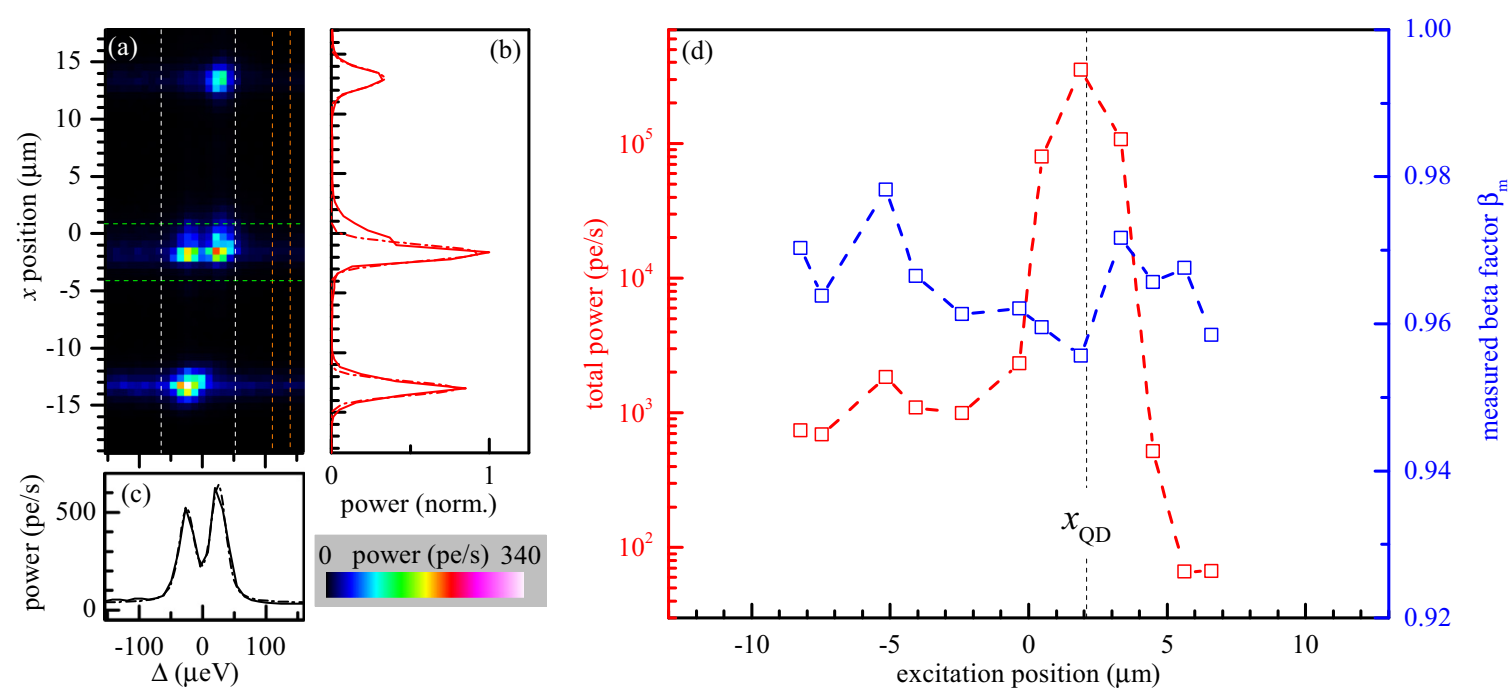

FIG. 5. Analysis of spectral images. (a) Spectral image of QD shown in Fig. 3(c), centered $1.400796 \mathrm{eV}$. (b) QD emission as a function of the position along the WG, taken as the spectral average around the QD emission energy [white dashed lines in (a)], and subtracting the background taken at a spectrally shifted region [orange dashed lines in (a)]. (c) Spectrum of the QD free-space emission (solid line), calculated as a spatial integral over the region given by the green dashed lines in (a); dashed dotted lines are a fit to the data. (d) Total power and beta factor of a QD as a function of the excitation position along the WG. The black dashed line indicates the position of the QD free-space emission.

other emitters, as exemplified by the white and orange dashed lines in Fig. 5(a), respectively. Phonon-assisted transitions of the QD are spectrally broad and provide a background about two orders of magnitude below the zero-phonon line emission, which can be neglected in this analysis. Subtracting the excitation background, we isolate the QD signal, as shown in Fig. 5(b), which is then fitted using three Gaussian peaks, to determine the position of the two couplers and of the QD free-space emission $x_{\mathrm{QD}}$. The $x$ axis is calibrated using the known distance between the couplers. The position error resulting from the fit is typically around $100 \mathrm{~nm}$ (standard deviation). Similarly, by fitting the background from other emitters, we determine the excitation position along the WG.

\section{APPENDIX B: BETA FACTOR VERSUS EXCITATION POSITION}

In Fig. 5(d) we show the total SE and the beta factor as a function of the excitation position along the PCWG for one specific QD. The total SE is the sum of the SE emitted from the bottom and top couplers and from the QD position into free space, as in Fig. 3(f). The total SE varies over three orders of magnitude for different excitation positions along the WG, showing a maximum at the QD location $x_{\mathrm{QD}}$ determined from the free-space SE as described in Appendix A. The beta factor determined versus excitation position also shown in Fig. 5(d) is constant within $1 \%$ standard deviation. This shows that the determination of the beta factor is robust against changes of excitation conditions. This variation is taken as the statistical error of $\beta_{\mathrm{m}}$. We note that out of the 31 analyzed QDs, only a few show significant nonlocal excitation and all of these emit between 1.393 and $1.377 \mathrm{eV}$. Nonlocal excitation is attributed to reabsorption of wetting layer emission into a WG mode. We can speculate that the QD absorption occurs into the trion $p$ shell (see Supplemental Material of [10,41]), which is about $40 \mathrm{meV}$ above the $s$ shell, consistent with the energy separation between the wetting layer emission around $1.42 \mathrm{eV}$ and the QD emission.

\section{APPENDIX C: QD EMISSION ENERGY AND WG MODE CIRCULARITY VERSUS POSITION}

One would expect that there is no correlation of energy and WG mode circularity with the QD position along the PCWG. The experimental data are given in Fig. 6, and exhibit a random distribution of energy and circularity along the QD position, consistent with this expectation.
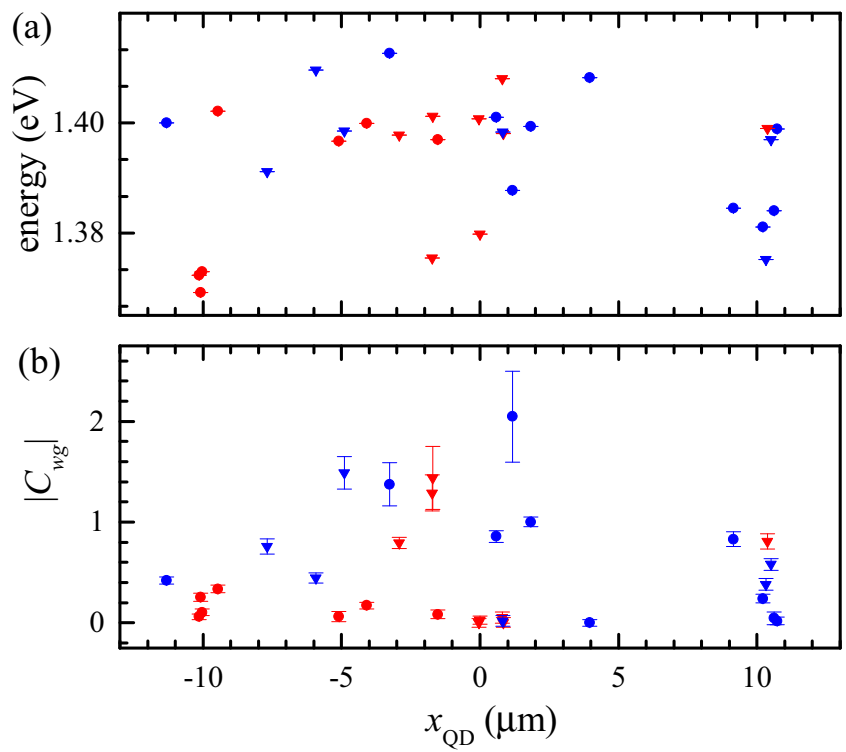

FIG. 6. Spatial distribution of (a) QD energy and (b) circularity along the WG, versus QD position $x_{\mathrm{QD}}$, determined as described in Appendix A. Symbols as in Fig. 4(d). 

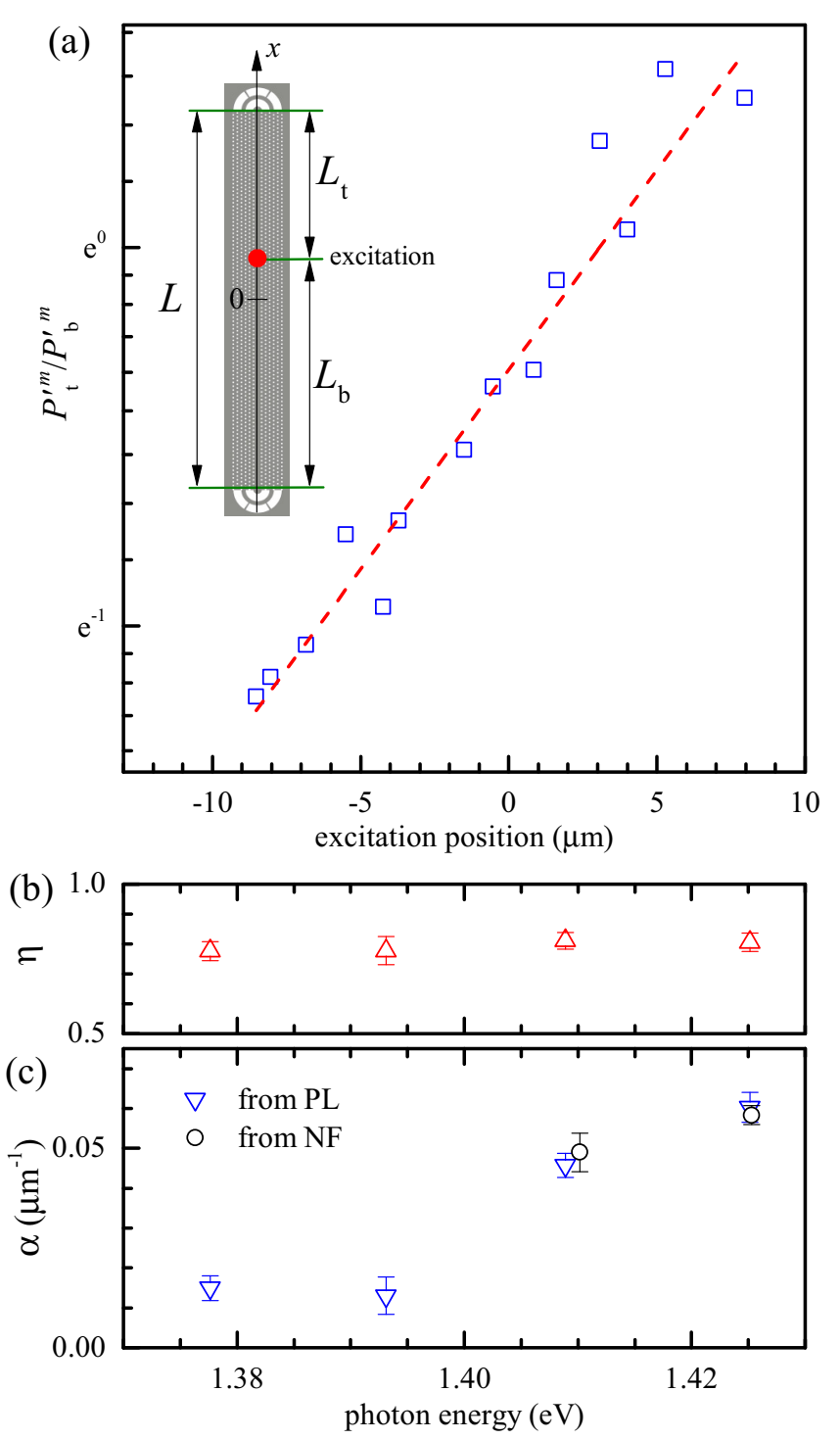

FIG. 7. PCWG loss determined by SE measurements. Analysis described in Appendix D applied to $\mathrm{WG}_{\mathrm{A}}$. (a) Top to bottom emission ratio $P_{\mathrm{t}}^{\prime \mathrm{m}} / P_{\mathrm{b}}^{\prime \mathrm{m}}$ as a function of the excitation position for the spectral range centered at $E_{\mathrm{L}}=1.425 \mathrm{eV}$. Inset: sketch of geometry. (b) Relative collection efficiency from top to bottom coupler versus center energy. (c) Loss coefficient $\alpha$ versus center energy determined from the coupler emission ratio (triangles) and NF imaging (circles).

\section{APPENDIX D: RELATIVE EFFICIENCIES OF COUPLERS}

To determine the relative efficiencies of the two couplers, we perform the following analysis on data taken with magnetic field. For a given excitation position along the PCWG, we define the distances $L_{\mathrm{t}}=L / 2-x$ and $L_{\mathrm{b}}=L / 2+x$ between the excitation position $x$ and the top and bottom couplers, respectively, with the length $L=26 \mu \mathrm{m}$ of the PCWG, as sketched in the inset of Fig. 7(a). Accounting for propagation losses $\alpha$, the SE powers emerging from the couplers are proportional to $\eta_{i} \exp \left(-\alpha L_{i}\right)$, with the efficiency $\eta_{i}$ of the couplers, where $i \in\{\mathrm{t}, \mathrm{b}\}$. Here, we assume that most of the emission occurs at the excitation position, consistent with the experimental findings of the imaging along the WG, as discussed in Appendix B. To apply the model, we spectrally integrate the SE from the bottom and top couplers over the range covered by the CCD camera $(\sim 15 \mathrm{meV})$, thereby averaging over the directionality of the individual QDs, resulting in the powers $P_{i}^{\prime m}$. The power ratio is fitted by

$$
\frac{P_{\mathrm{t}}^{\prime \mathrm{m}}}{P_{\mathrm{b}}^{\prime \mathrm{m}}}=\eta e^{2 \alpha x}
$$

with $\eta=\eta_{\mathrm{t}} / \eta_{\mathrm{b}}$, as shown in Fig. 7(a) for the spectral range centered at $E_{0}=1.425 \mathrm{eV}$ for $\mathrm{WG}_{\mathrm{A}}$. A good agreement between data and fit is observed. We repeated the analysis for the four spectral ranges considered in the experiment, and show in Figs. 7(b) and 7(c) the resulting $\eta$ and $\alpha$ versus $E_{\mathrm{L}}$. We find that $\eta$ has no significant dependence on $E_{\mathrm{L}}$, and we use the average value of $\eta=0.79 \pm 0.02$ to correct the emission intensities of all the data from this WG. The analysis has been repeated with $\mathrm{WG}_{\mathrm{B}}$, giving a similar result $\eta=0.62 \pm 0.02$. The loss $\alpha$ increases with $E_{\mathrm{L}}$, as expected from the increasing absorption by the wetting layer and the increasing radiation losses (see Appendix G 2). Notably, the values are consistent with the loss measured by NF imaging, shown as black circles in Fig. 7(c).

The determined $\eta$ and $\alpha$ are used to calculate the powers $P_{\mathrm{b}}^{j}$ and $P_{\mathrm{t}}^{j}$ unaffected by loss and relative coupling efficiency, used in Eq. (2), as

$$
\begin{aligned}
& P_{\mathrm{b}}^{j}=P_{\mathrm{b}}^{j, \mathrm{~m}} \exp \left[\alpha\left(x_{\mathrm{QD}}+L / 2\right)\right], \\
& P_{\mathrm{t}}^{j}=\eta^{-1} P_{\mathrm{t}}^{j, \mathrm{~m}} \exp \left[\alpha\left(L / 2-x_{\mathrm{QD}}\right)\right] .
\end{aligned}
$$

\section{APPENDIX E: BACK REFLECTIONS AT PCWG TERMINATION}

Back reflections at the couplers can affect the observed circularity since light emitted in a given direction is detected after the reflection at the opposite coupler. To estimate the influence of this effect for the investigated sample, we consider the measured powers of the QD with the highest mode circularity we found. To first order in the reflection coefficient $R$, the reflection of the measured power from the bottom coupler is adding to the measured power from top coupler, and vice versa. The powers unaffected by the reflection are then given by $\tilde{P}_{\mathrm{t}}^{j} \approx P_{\mathrm{t}}^{j, \mathrm{~m}}-R P_{\mathrm{b}}^{j, \mathrm{~m}}$, and $\tilde{P}_{\mathrm{b}}^{j} \approx P_{\mathrm{b}}^{j, \mathrm{~m}}-R P_{\mathrm{t}}^{j, \mathrm{~m}}$. Requiring that these powers are positive, we find from the measured powers of all investigated QDs shown in Fig. 3 an upper limit for $R$ of about $3 \%-5 \%$. The reflection leads to a systematic underestimation of the absolute circularity and directionality, and for $5 \%$ is limiting the measured directionality to $\left|D_{\mathrm{m}}\right|<0.95$ and the measured circularity to $\left|C_{\mathrm{m}}\right|<1.8$. The dynamic range of our experimental data is sufficient to measure $\left|C_{\mathrm{m}}\right|$ above 4 , and $\left|D_{\mathrm{m}}\right|$ above $99.9 \%$, and is thus not limiting the results presented.

\section{APPENDIX F: NF AND FF ANALYSIS}

In Fig. 2(b) in the main text, we show an example of the NF of the radiation losses along the waveguide. The data were obtained using the sum of the 100 subsequent frames of a video taken by a Sony DCR-TRV620E digital 8 camcorder in nightshot mode. The dark background was subtracted for all data shown. The NF emission has been derived as the difference between the emission profile along the waveguide 


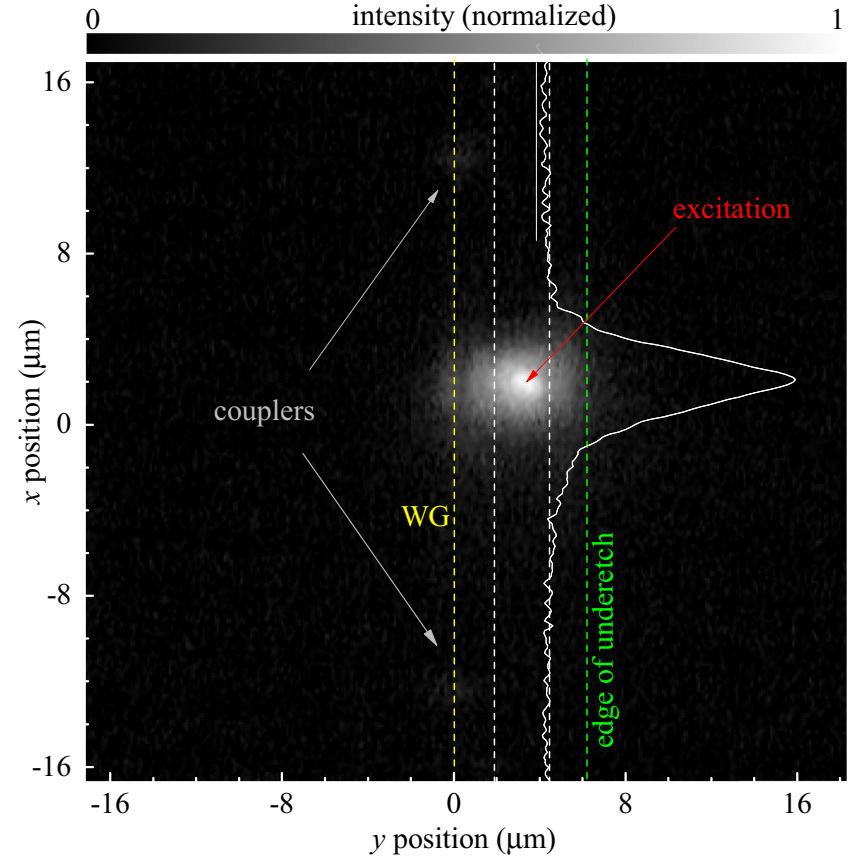

FIG. 8. Carrier diffusion in the GaAs membrane. PL emission image for excitation on the PC membrane as indicated. Green dashed line: edge of underetched region. Carriers reaching the WG (yellow dashed line) excite the QDs and wetting layer, and emission is visible from the couplers. Solid white line: average intensity between white dashed lines, with zero given by baseline

and a background profile next to the waveguide. Each profile is averaged over $1.7 \mu \mathrm{m}$ in the $x$ direction, as indicated in Fig. 2(b) with white and red dashed lines, respectively. The $x$ axis is calibrated using the known distance between the couplers. We repeated the analysis for different excitation energies, and calculated the corresponding loss coefficients. The result is shown in the inset of Fig. 2(a). The origin of these losses is discussed in Appendix G 2 .

\section{Carrier diffusion length}

The emission imaging allows to measure the carrier diffusion length on the photonic crystal membrane. In particular, we image the PL emission from the QDs for excitation about $3 \mu \mathrm{m}$ sideways offset from WG, on the unstructured freestanding membrane, as shown in Fig. 8. The spatially resolved emission is extended compared to the excitation, due to carrier diffusion between excitation and recombination. In order to evaluate the diffusion length, we average over about $2.5 \mu \mathrm{m}$ in the $y$ direction (see white dashed lines). The corresponding profile, given as solid white line, is fitted with a Gaussian profile, showing a standard deviation of about $1.4 \mu \mathrm{m}$, which represents the carrier diffusion length along the $x$ direction. Some of the carriers diffuse into the WG region, exciting QDs that couple to the guided mode, as is evident from the weak emission observed from the couplers.

\section{Far-field width}

The far-field profile [see Fig. 2(c)] has a finite FWHM, $\gamma_{\mathrm{FF}}$, in the $k_{x}$ direction. There are two contributions to the
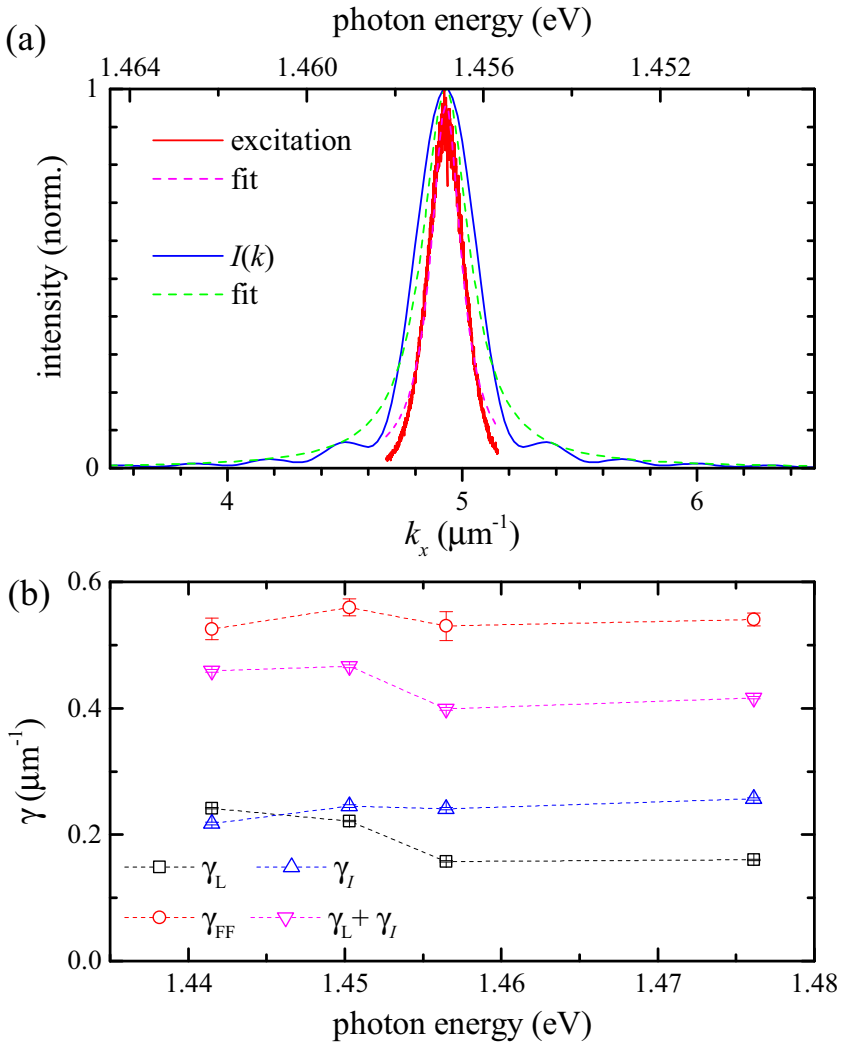

FIG. 9. (a) Solid lines: excitation spectrum and $I(k)$; dashed lines: corresponding Lorentzian fits. (b) FF width $\gamma_{\mathrm{FF}}$ in the $k_{x}$ direction interpreted as the sum of the excitation width $\gamma_{\mathrm{L}}$ and the width $\gamma_{I}$ of $I(k)$. All widths are the FWHM of Lorentzian fits.

observed width: (i) the spectral width of the excitation laser, which translates via the PCWG dispersion into a FWHM $\gamma_{\mathrm{L}}$, and (ii) the spatial profile of the NF in the $x$ direction, which is given by an exponential decay of the field amplitude due to losses, delimited by the aperture length $L_{\mathrm{p}}$. The observed field amplitude is thus modeled, neglecting constant factors, as

$$
E(x)=\left[\theta\left(x+\frac{L_{\mathrm{p}}}{2}\right)-\theta\left(x-\frac{L_{\mathrm{p}}}{2}\right)\right] \exp \left(-\frac{\alpha x}{2}\right),
$$

with the Heaviside function $\theta$. Fourier transforming and taking the absolute square, we find the corresponding intensity in $k$ space is given by

$$
I(k)=\frac{2}{k^{2}+\frac{\alpha^{2}}{4}}\left[\cosh \left(\frac{\alpha}{2} L_{\mathrm{p}}\right)-\cos \left(k L_{\mathrm{p}}\right)\right] .
$$

For large losses across the aperture, $\alpha L_{\mathrm{p}} \gg 1$, the constant cosh term dominates and $I(k)$ is a Lorentzian, while for small losses, $\alpha L_{\mathrm{p}} \ll 1$, the cos term dominates, resulting in a sinc function. We fit Eq. (F2) with a Lorentzian to determine the equivalent FWHM $\gamma_{I}$. The laser spectrum, converted into $k_{x}$ using the linear dispersion of about $-27.3 \mu \mathrm{m}^{-1} \mathrm{eV}^{-1}$ in the relevant range (see Fig. 2), and $I(k)$, together with the corresponding Lorentzian fits to determine $\gamma_{\mathrm{L}}$ and $\gamma_{I}$, respectively, are shown in Fig. 9(a) for $E_{\mathrm{L}}=1.4565 \mathrm{eV}$, as used in Figs. 2(b) and 2(c). The FF profile is then given by the convolution of (i) and (ii), which for Lorentzians keeps a Lorentzian, having a width given by the sum of the widths, 
here $\gamma_{L}+\gamma_{I}$. The result of this analysis is shown in Fig. 9(b) for all measured $E_{\mathrm{L}}$. Generally a good quantitative agreement is found. The remaining difference between $\gamma_{\mathrm{L}}+\gamma_{I}$ and $\gamma_{\mathrm{FF}}$ of about $0.1 \mu \mathrm{m}^{-1}$ could be related to a slight defocus of the FF imaging.

\section{Calibration of FF imaging}

In order to calibrate the in-plane wave vector in the FF imaging, we measured the FF of the emission of the top coupler when exciting into the bottom coupler at $E_{\mathrm{L}}=1.4102 \mathrm{eV}$, as shown in Fig. 10(a). To use the known NA of the objective for calibration, we determine the cutoff radius, using the polar coordinate representation of the data, as shown in Fig. 10(b). We adjusted the center of the coordinate system to obtain a constant maximum radius, shown by the vertical dashed line. We average the $\theta$ range between the two horizontal dashed lines in Fig. 10(b), obtaining the profile shown in Fig. 10(c). We take the NA radius to be the value of $k$ at half step height, and calibrate this radius to $k_{0} \mathrm{NA}=6.076 \mu \mathrm{m}^{-1}$. A relative error of a few $\%$ rms of this calibration is estimated.

\section{APPENDIX G: SIMULATION METHODS}

\section{Geometry and parameters}

FDTD calculations using the package MEEP [42] were carried out to model the properties of the PCWGs under study. All calculations used a resolution of 24 points per lattice constant, and a cubic Yee lattice. The permittivity $\varepsilon$ of GaAs at $T=5 \mathrm{~K}$ including its dispersion [37] is given by

$$
\begin{aligned}
\varepsilon(E)= & 5.965 \\
& +\frac{0.0304}{1.519^{2}-E^{2}}+\frac{33.1494}{2.692^{2}-E^{2}}+\frac{0.00238}{0.0334^{2}-E^{2}},
\end{aligned}
$$

with the photon energy $E$ in units of $\mathrm{eV}$. This permittivity was implemented in the FDTD dispersion model as shown in Fig. 11 over the relevant energy range.

Single unit-cell simulations were carried out with periodic boundary conditions along $x$ and a current source at $z=$ 0 and a given position in $x, y$. Initially, these calculations made use of the nominal sample parameters: lattice constant $a=260 \mathrm{~nm}$, hole radius $r=0.24 a$, and slab height $h=$ $0.4808 a=125 \mathrm{~nm}$. Perfectly matched layers (PMLs) were placed on the out-of-plane simulation facets at $\pm z_{\mathrm{s}}$ and adiabatic absorbers [43] on the in-plane facets at $\pm y_{\mathrm{s}}$ to absorb light tunneling through the cladding. Six layers of air holes were placed either side of the waveguide, matching the sample. A sketch of the geometry covering one unit cell of the PCWG is shown in Fig. 12.

To calculate the WG mode dispersion, we implemented periodic boundary conditions with a phase factor over a single PCWG unit cell, and extracted the mode resonances from the time-domain Fourier transforms as function of the phase.

Simulations with the nominal structural parameters showed significant deviations from the measured dispersion. As discussed in the main text, a single parameter $d$ was introduced, describing the thickness of material removed from all surfaces of the structure, for example, by etching during fabrication or
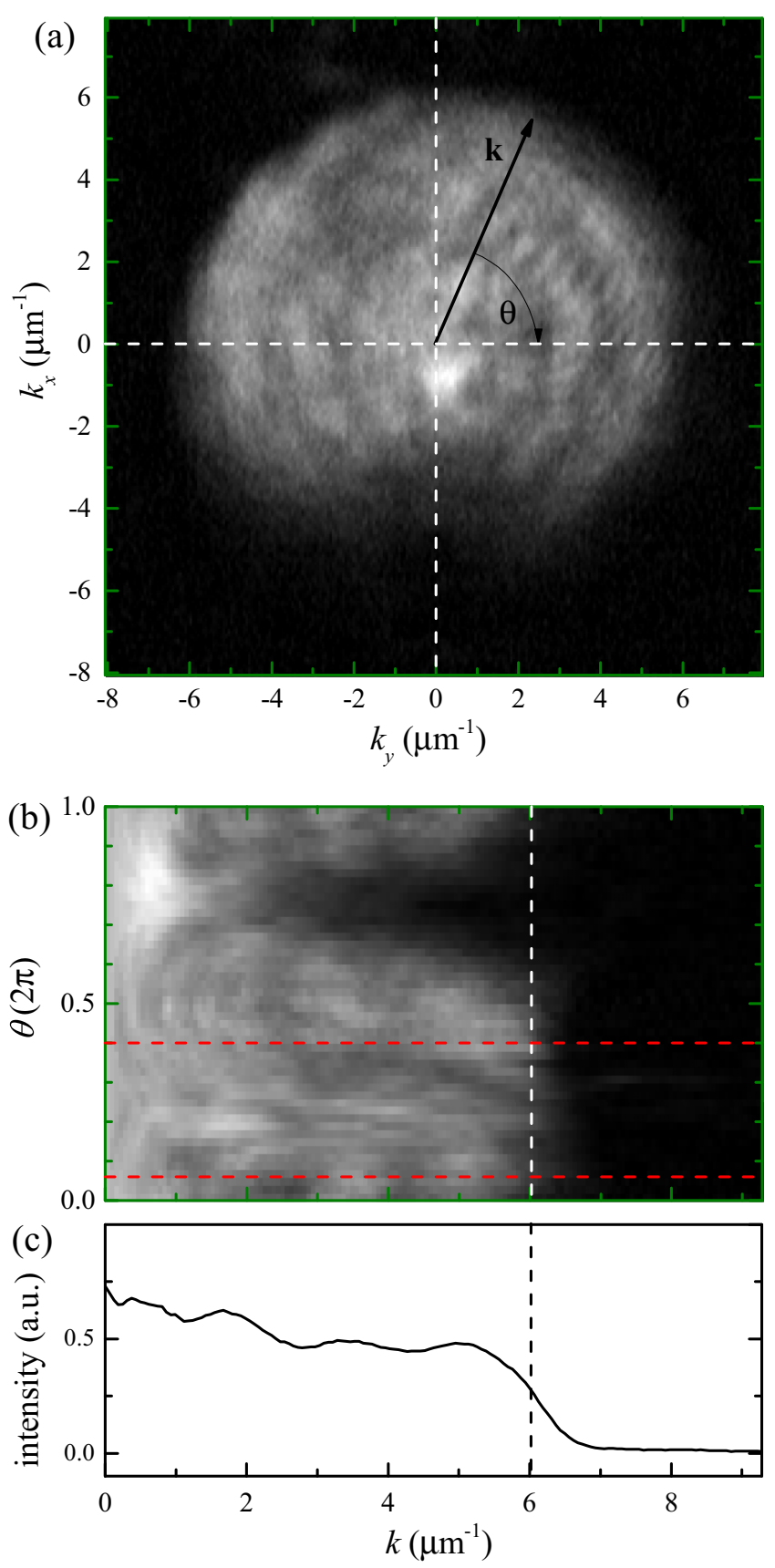

FIG. 10. NA calibration of FF imaging. (a) Emission from top coupler for excitation into the bottom coupler at $E_{\mathrm{L}}=1.4102 \mathrm{eV}$. (b) Polar representation of (a). (c) Radial profile of (b) averaged over the $\theta$ range indicated by horizontal dashed lines in (b). Vertical dashed lines in (b) and (c) indicate the radius of the NA in $k$ space. Grayscale as in Fig. 8.

subsequent oxidation. Simulations were carried out with the radius of the holes expanded by $d$, and the height of the slab reduced by $2 d$. We found that $d$ around 7-8 nm produces a good match with experiment (see Fig. 13), and used $d=8 \mathrm{~nm}$ for the remaining calculations, for both investigated WGs.

\section{PCWG loss}

A significant number of QDs measured are in resonance with WG modes with propagation wave vectors $k_{x}$ within the 


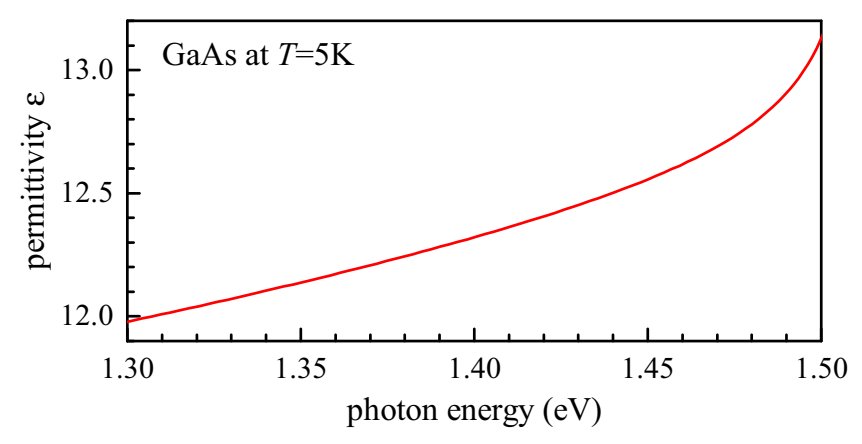

FIG. 11. GaAs permittivity $\varepsilon$ as function of photon energy used in the calculations. Data from the material implementation of Eq. (G1) in FDTD.

light cone, $k_{x}<2 \pi v c / a$ with the mode eigenfrequency $v$, and the speed of light $c$. Modes in this part of the dispersion emit into free space and thus experience propagation loss, even in an ideal structure. In addition to this out-of-plane loss, all modes will experience an in-plane loss due to the finite extension of the lateral PC, allowing light to tunnel through the PC cladding either side of the WG.

The loss was simulated with the method described in Appendix G 1, choosing the symmetry of the simulation and sources either even or odd to excite only one of the two WG modes (see Appendix G3). The simulation geometry is as sketched in Fig. 12, with the height extended to $10 a$ and the PML thickness to $1.5 a$, in order to allow for better separation of the out-of-plane loss from the in-plane propagation. We use a source with a Gaussian time dependence of 29 fs standard deviation, truncated at 5 standard deviations either side of the peak. To maximize excitation of the WG mode of interest, the source center frequency was set to the WG mode frequency at the simulated $k_{x}$, as calculated in Appendix G 1. The simulation was run for 1000 time steps, ending about $870 \mathrm{fs}$ after the source is switched off, at which point the remaining field of nonguided modes is negligible, as is evidenced by a stable field distribution, apart from a global oscillating and decaying prefactor $\exp (-i \omega t)$ with the complex mode frequency $\omega\left(k_{x}\right)$. The Poynting vector of this field was then evaluated, providing the power flux density of the selected WG mode. This distribution was used to calculate the flux $F_{x}$ through the $+x_{\mathrm{s}}$ plane, representing the propagating flux
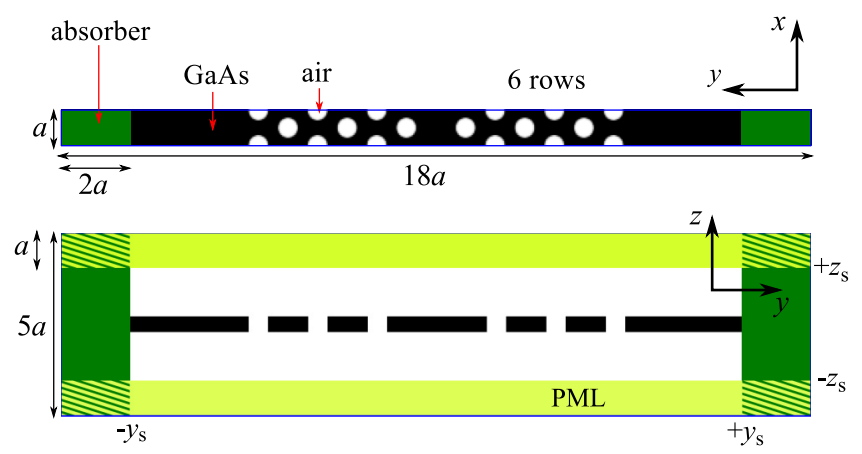

FIG. 12. Sketch of the simulation volume used in the band structure calculations, covering one unit cell of the PCWG in $x$ direction.
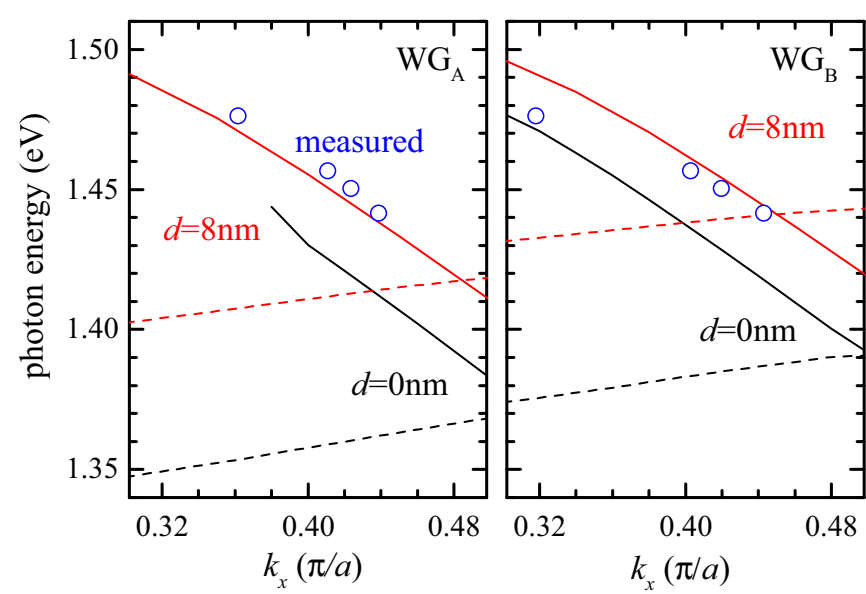

FIG. 13. Matching simulations to the measured mode dispersion for $\mathrm{WG}_{\mathrm{A}}$ (left) and $\mathrm{WG}_{\mathrm{B}}$ (right). Solid lines: fundamental mode; dashed lines: higher-order mode; black lines: $d=0 \mathrm{~nm}$; red lines: $d=8 \mathrm{~nm}$; circles: measured (see Fig. 2).

along the PCWG, and the flux $F_{z}$ through the $\pm z_{\mathrm{s}}$ planes, representing the out-of-plane loss, and $F_{y}$ through the $\pm y_{\mathrm{s}}$ planes, representing the in-plane loss. The transmission coefficient per unit cell is then given by $T=F_{x} /\left(F_{z}+F_{y}+F_{x}\right)$, from which the loss coefficient $\alpha=-\ln (T) / a$ is determined, as shown in Fig. 14 for the two WG modes as a function of energy. The loss coefficient considering in-plane loss only (using $F_{z}=0$ ) is also given. The even (higher-order) mode has a dispersion showing a maximum, and thus presents two $k$ vectors for a given energy over a significant range. One branch corresponds to the part of the even mode inside the light cone, showing high loss, dominated by out-of-plane loss, while the other is outside the light cone and shows only the small remaining in-plane loss due to the tunneling through the

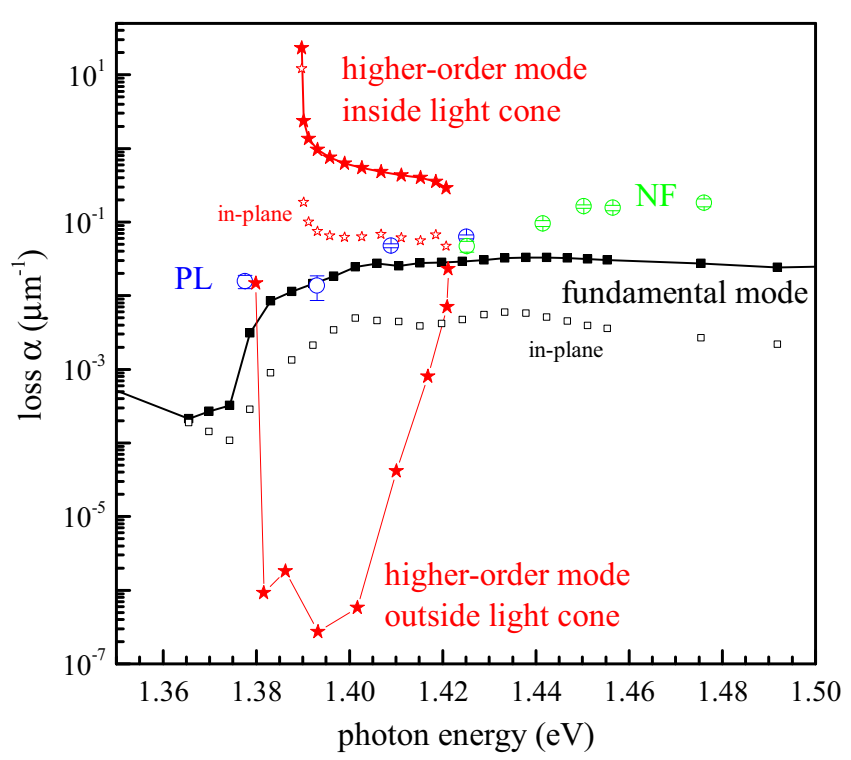

FIG. 14. Simulated propagation loss of the fundamental mode (filled black squares) and higher-order mode (filled red stars). The inplane losses are given as empty symbols. Blue circles: loss measured from PL. Green circles: loss measured from NF. 
fundamental mode $k_{x}=0.54 \pi / a$
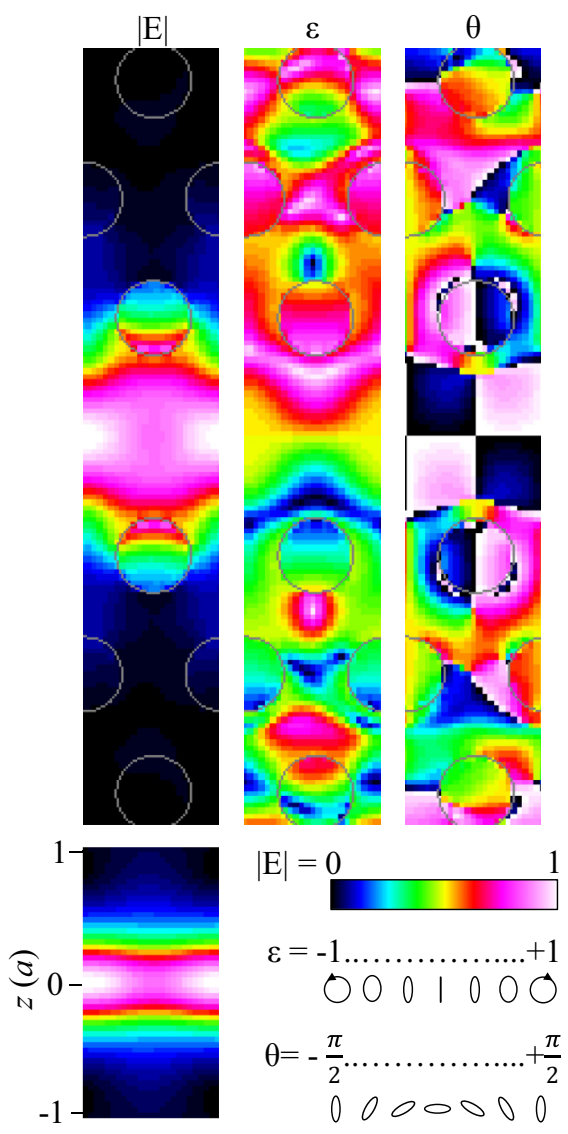

higher order mode $k_{x}=0.05 \pi / a$
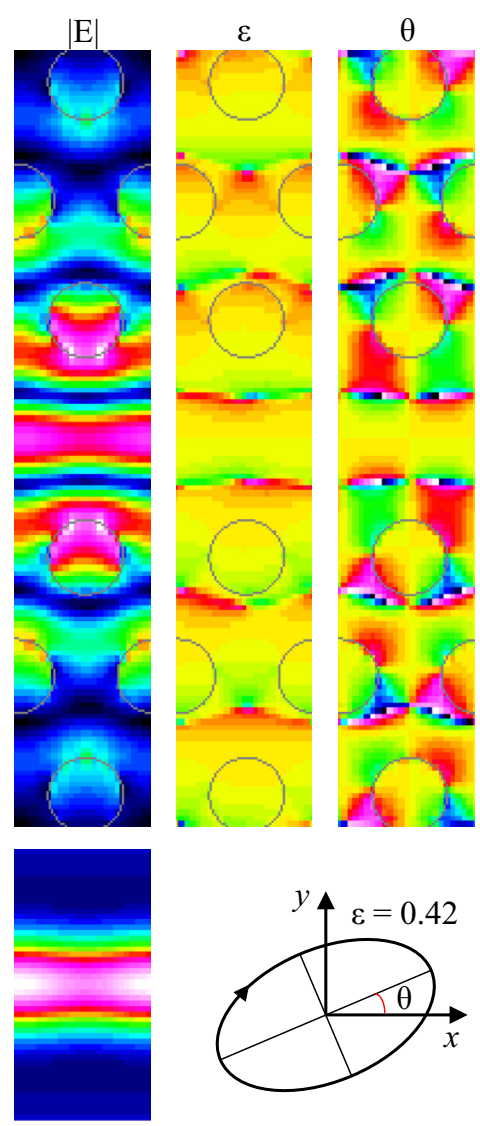

higher order mode $k_{x}=0.86 \pi / a$
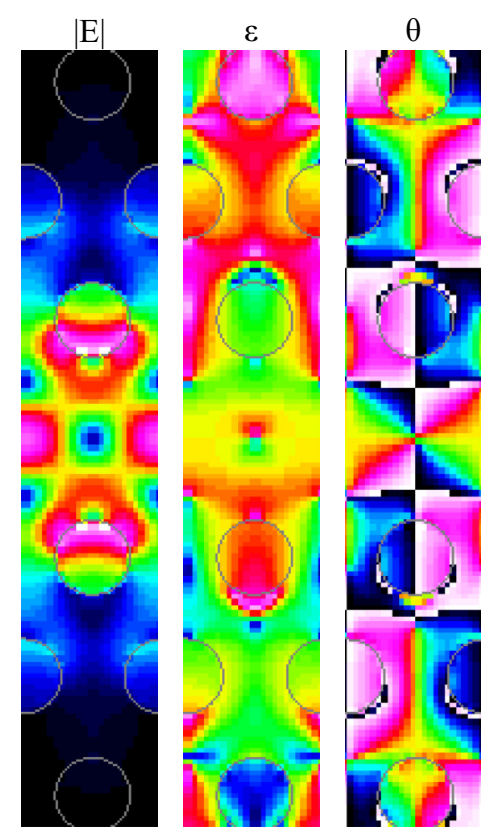
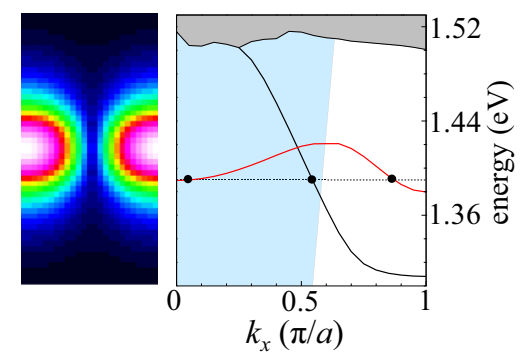

FIG. 15. Absolute value of the electric field amplitude $|E|$, polarization ellipticity $\varepsilon$, and orientation $\theta$, in the plane $z=0$, for $\mathrm{WG}_{\mathrm{A}}$. The hole structure is superimposed for reference. $\theta$ is defined according to the sketch shown in the central panel. The source energy is $E=1.39 \mathrm{eV}$, which corresponds to the indicated propagation wave vectors, according to the inset in the right panel, which are imposed by the phase of the boundary condition. $x z$ sections of $|E|$ at $y=0$ are shown below the corresponding $x y$ sections.

cladding. The odd (fundamental) mode shows increasing loss with increasing energy. Below $1.38 \mathrm{eV}$, the mode is outside the light cone, so that the loss is strongly reduced to the in-plane loss only. The measured loss is indicated by the circular data points, and shows a good agreement with the calculated loss of the odd mode. The additional loss in the measurements above $1.43 \mathrm{eV}$ is attributed to absorption in the wetting layer, not taken into account in the simulations.

From the same simulations we also obtain the mode field amplitude and polarization. In Fig. 15, the absolute value of the electric field amplitude $|E|$, the ellipticity $\varepsilon$, and the orientation $\theta$ of the polarization ellipse are shown in the plane $z=0$, for $\mathrm{WG}_{\mathrm{A}}$. Note that for $z=0$ the $z$-field component is zero. Orientation and ellipticity are defined as

$$
\begin{aligned}
\theta & =\frac{1}{2} \arctan \left(\frac{S_{2}}{S_{1}}\right), \\
|\varepsilon| & =\sqrt{\frac{2}{\sqrt{S_{1}^{2}+S_{2}^{2}}+1}-1,} \\
\varepsilon & =|\varepsilon| \operatorname{sign}\left(S_{3}\right),
\end{aligned}
$$

with the Stokes parameters $S_{1}=\left(\left|E_{x}\right|^{2}-\left|E_{y}\right|^{2}\right) / S_{0}, S_{2}=$ $2 \operatorname{Re}\left(E_{x}^{*} E_{y}\right) / S_{0}$, and $S_{3}=2 \operatorname{Im}\left(E_{x}^{*} E_{y}\right) / S_{0}$ and $S_{0}=\left|E_{x}\right|^{2}+$ $\left|E_{y}\right|^{2}$. We can clearly see the spatial extension of the modes in their amplitude, and their circular and linear points in their ellipticity. The fundamental mode is linear in the center (yellow in $\varepsilon$ ), and circular of opposite helicity (black and white) in the center of the unit cell along $x$, close to the first holes. Other linear and circular points are present, but at much reduced mode field amplitudes. The higher-order mode at a small $k_{x}=0.05 \pi / a$ is dominated by linear polarization, and at large $k_{x}=0.86 \pi / a$ has circular points at the edge of the unit cell, at lower field strengths. This is a consequence of the temporal symmetry that ensures that no component of circular polarization can exist at the band edge points $k_{x}=0$ and $k_{x}= \pm \pi / a$ in this type of waveguide [44]. Thus, in the proximity of these $k_{x}$ one finds either a reduced component of circular polarization and/or a reduction in the electric field strength in the regions with circular polarization.

To give some insight into the radiative loss of the modes, 2D spatial Fourier transforms were carried out on the fields of the guided modes. The fields in the $z=0$ plane of the slab were used. In this plane, symmetry ensures that the three 

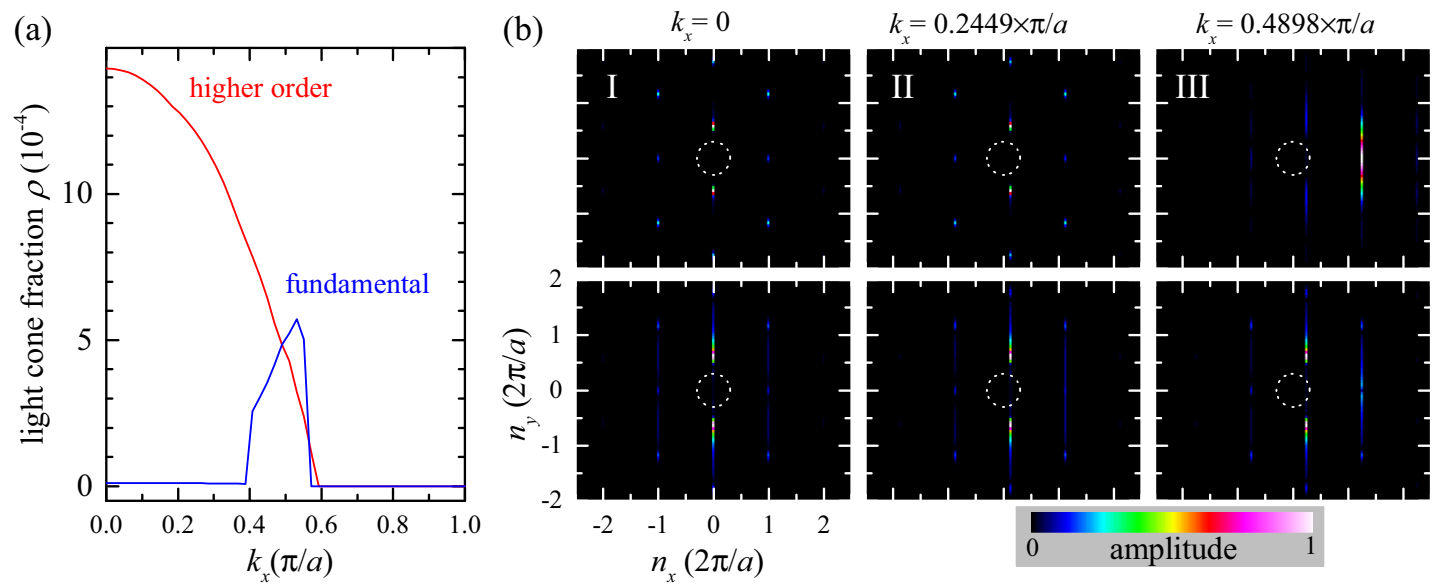

$k_{x}=0.73470 \times \pi / a$
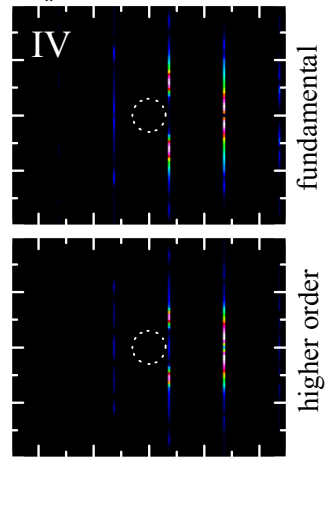

FIG. 16. Fourier analysis of WG mode fields. (a) Fraction $\rho$ of intensity $\left|\tilde{H}_{z}(\mathbf{n})\right|^{2}$ inside the light cone $\mathcal{L}$, for the fundamental (blue line) and the higher-order (red line) mode, versus Bloch wave vector $k_{x}$. (b) $\left|\tilde{H}_{z}(\mathbf{n})\right|$ of the fundamental (upper panels) and higher-order (lower panels) modes at specific $k_{x}$ as indicated. The white circles represent the light cone $\mathcal{L}$.

nonzero field components are $E_{x}(\boldsymbol{r}), E_{y}(\boldsymbol{r})$, and $H_{z}(\boldsymbol{r})$. The profile of $\left|H_{z}(\boldsymbol{r})\right|^{2}$ appears nearly identical to $\left|E_{x}(\boldsymbol{r})\right|^{2}+\left|E_{y}(\boldsymbol{r})\right|^{2}$, and we choose to use for the following discussion $\tilde{H}_{z}(\mathbf{n})$, the Fourier transform with wave vector $\mathbf{n}$. Components at $|\mathbf{n}|<$ $k_{0}$, inside the light cone $\mathcal{L}$, can couple to free-space modes, having an intensity fraction $\rho=\left[\int_{\mathcal{L}} \xi(\mathbf{n}) d \mathbf{n}\right] /\left[\int \xi(\mathbf{n}) d \mathbf{n}\right]$, with $\xi(\mathbf{n})=\left|\tilde{H}_{z}(\mathbf{n})\right|^{2}+\left|E_{x}(\mathbf{n})\right|^{2}+\left|E_{y}(\mathbf{n})\right|^{2}$, which is indicative of the loss rate per unit time [45]. For this calculation, the mode fields were extracted from an eigensolver [46], using $\varepsilon=12.25$, and $d=0 \mathrm{~nm}$. The fraction $\rho$ is given in Fig. 16(a) for both WG modes as function of the Bloch wave vector $k_{x}$, and the distribution of $\left|\tilde{H}_{z}(\mathbf{n})\right|$ is shown in Fig. 16(b) for selected $k_{x}$.

The fundamental mode for small $k_{x}$ [see Fig. 16(b), panels I and II] is in the continuum above the band gap of the PC cladding, and thus able to propagate in the $\pm y$ directions, leading to a small extension in $n_{y}$. This concentration reduces the overlap with the light cone, hence the low $\rho$ in Fig. 16(a) in this region. For higher $k_{x}$ (see panel III) the mode enters the band gap, confining it in $y$ and thus broadening it in $n_{y}$. This results in an increased $\rho$, until $k_{x}$ leaves the light cone (panel IV). In contrast, the higher-order mode [see bottom row of Fig. 16(b)] remains inside the band gap for all $k_{x}$, so that $\rho$ is decreasing monotonically with increasing $k_{x}$, reaching zero at the edge of the light cone, $k_{x}=k_{0}$.

In order to estimate the propagation loss coefficient $\alpha_{\text {FT }}$ per unit distance, the fraction $\rho$ is scaled as

$$
\alpha_{\mathrm{FT}}=\frac{1}{v_{\mathrm{g}}} \frac{c k_{z}}{h k_{0}} \rho .
$$

In this expression, the factor $\left(c k_{z}\right) /\left(h k_{0}\right)$ is the attempt rate with which the light is emitted, with the emission fraction $\rho$ per attempt. The factor $1 / v_{\mathrm{g}}$, with the group velocity $v_{\mathrm{g}}$, converts the resulting loss per unit time into the loss per unit propagation length. The resulting loss is given in Fig. 17. This approach only accounts for out-of-plane losses and assumes an infinite WG without absorption and disorder. The loss thus becomes exactly zero outside the light cone, beyond the lower end of the curves in Fig. 17. Notably, this estimate of loss is qualitatively reproducing the one calculated via FDTD (see Fig. 14) for both modes, and is quantitatively about a factor of 3 lower.

\section{Coupler efficiency}

FDTD simulations were used to determine the efficiency of the couplers. A sketch of the simulation volume used is shown in Fig. 18. In order to calculate the efficiency and reflectivity of the coupler, two simulations are used. The coupler simulation contains the actual structure, and a calibration simulation is identical except that the coupler is removed and replaced by a continuation of the PCWG. The power in the PCWG mode traveling toward the coupler, called the input power $P_{\mathrm{i}}$, is determined by the calibration simulation, given by the power through the flux plane indicated in blue in Fig. 18. After determining the power $P_{\text {ic }}$ through the same flux plane in the coupler simulation, the coupler reflectivity is calculated as $R_{\mathrm{c}}=1-P_{\mathrm{ic}} / P_{\mathrm{i}}$. Using the power radiated out of the coupler $P_{\mathrm{ec}}$ through the flux plane indicated in red in Fig. 18, located $a / 20=13 \mathrm{~nm}$ above the slab, the coupler efficiency is calculated as $\eta_{\mathrm{c}}=P_{\mathrm{ec}} / P_{\mathrm{i}}$. Note that due to the inversion symmetry in $z$, the same power is emitted from either side toward both sides of the slab WG.

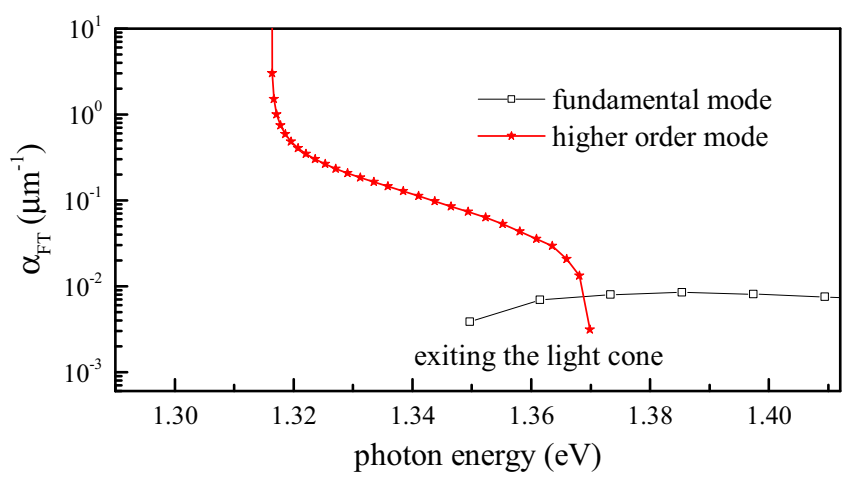

FIG. 17. Estimated loss $\alpha_{\mathrm{FT}}$ of the two WG modes using Eq. (G3) based on the fraction $\rho$ inside the light cone, shown in Fig. 16. 


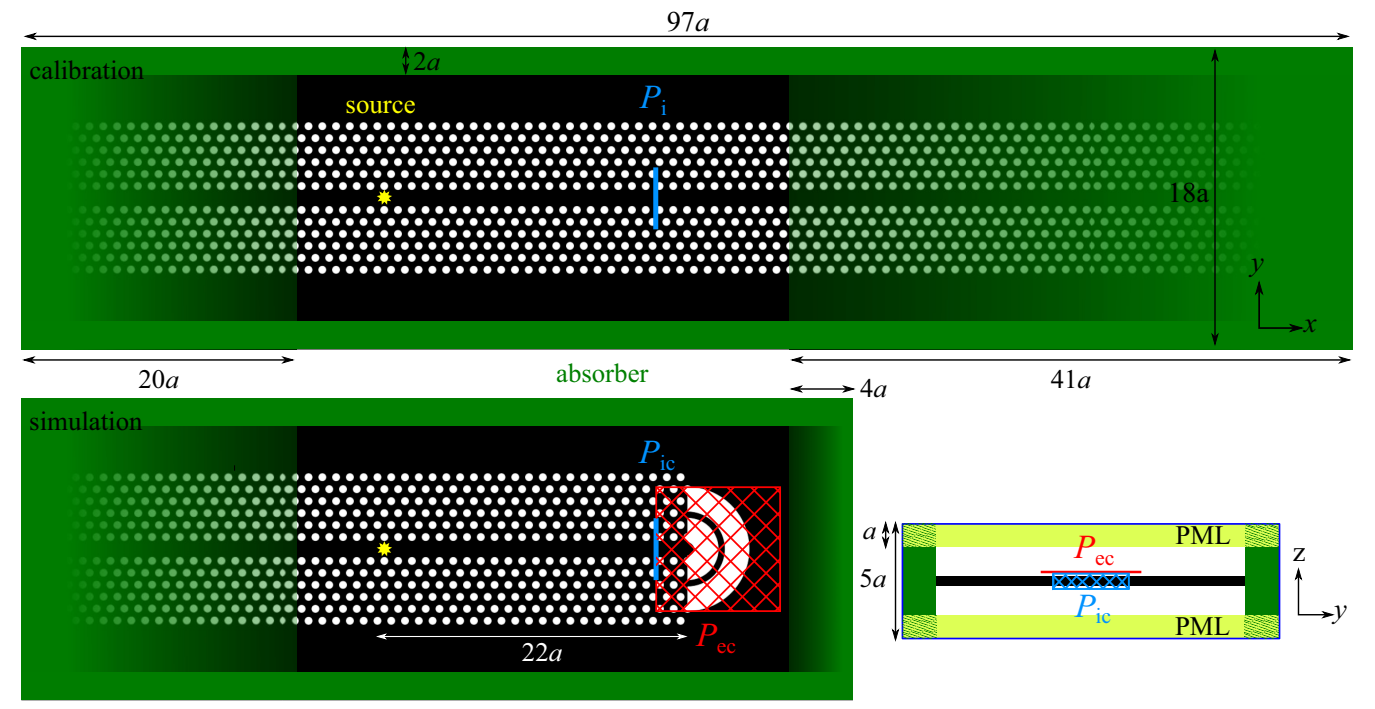

FIG. 18. Sketch of the FDTD simulation volumes used to determine coupler efficiency and reflectivity, consisting of a calibration simulation (top) and a coupler simulation (bottom). The green areas indicate absorbers. The blue line indicates the plane that measures the powers $P_{\mathrm{i}}$ and $P_{\mathrm{ic}}$. The red hatched region indicates the area over which the power emitted by the coupler is measured. On the bottom right, a view along the WG is given, stretched by $20 \%$ along $z$ for clarity. For the even-mode calculations, the simulation volume is expanded by $5 a$ on each $x$ facet. The radial bars supporting the grating were not included in the simulation.

In the simulations, an electrical dipole source (yellow star in Fig. 18) with a time-varying current given by $\left[j_{x}(t), j_{y}(t)\right] \propto \mathbf{d} \exp \left[-i \omega t-t^{2} /\left(2 \Delta_{t}^{2}\right)\right]$ is used, with the time $t$, the central frequency $\omega$, the duration $\Delta_{t}$, and the dipole vector d. Note that MEEP implements such a source using discrete time derivatives of Gaussians for better performance. The source frequency was set to $\omega=1.8395 c / a$, at the center of the measured QD distribution $(\hbar \omega \approx 1.3936 \mathrm{eV})$. The source has a duration of $\Delta_{t}=50 \mathrm{a} / \mathrm{c} \approx 43 \mathrm{fs}$ standard deviation, which results in a frequency standard deviation of $\Delta_{\omega}=2 \pi / \Delta_{t} \approx 10 \mathrm{meV} / \hbar$, covering the range of QD energies measured experimentally. To convert the simulated fields from time domain to frequency domain, we use MEEP's fluxplane function [42].

The source and simulation symmetry were chosen to select for either even or odd modes. For the odd mode, the source was polarized along the $y$ direction $(\mathbf{d}=[0,1])$, and placed at coordinates $[0.057,0] a$ relative to the center of the unit cell (see Fig. 15). For the even mode, two in-phase $x$-polarized sources are used $(\mathbf{d}=[1,0])$ placed at $[0.057, \pm 0.31] a$. The symmetries were exploited to gain a factor of 4 reduction in simulation time and memory, using an even mirror plane at $z=0$, and a mirror plane at $y=0$ with a parity matching the simulated mode [42].

The simulation size is $97 a$ in $x, 18 a$ in $y$, and $5 a$ in $z$ direction, as shown in Fig. 18. Thick adiabatic absorbers covering the reflecting simulation boundaries in $x$ direction were found to be necessary since PCWG modes, specifically at low group velocity, are easily reflected from the spatially varying absorption in the absorbers. The absorbers are implemented via an electric and magnetic conductivity, and we used a scaling of this conductivity proportional to the sixth power of the depth into the absorber, which provided lower reflections compared to using second and fourth power. For the odd mode, in the calibration simulation, the absorber thickness in the positive $x$ direction was set to $41 a$. The negative $x$ direction simulation boundary is less critical, as any reflections from this boundary will match between the calibration and coupler simulations, so that only the weaker reflection of the coupler in the coupler simulation needs to be sufficiently suppressed. We used a thickness of $20 a$. The other simulation facets do not have photonic structures intersecting them, and thus can be treated with shorter absorbers. We used $2 a$ in the $\pm y$ directions, and PMLs of thickness $a$ in $z$. In the coupler simulation, there is no photonic lattice at the $+x$ boundary, and an absorber of $4 a$ thickness was used. The frequency window of interest includes slow group velocity regions of the even mode, making them even more sensitive to being reflected by absorbers. In the even-mode simulations, the absorber thickness on the $\pm x$ boundaries were increased by $5 a$. The absorber strength was set to $\frac{1}{2}\left(\frac{1}{18}\right)$ of MEEP's default [42], for the odd (even) mode, respectively. We found that $P_{\mathrm{i}}$ varied by about $0.7 \%$ in the calibration simulations changing the absorber thickness on the $\pm x$ facets to $15 a$. Furthermore, displacing the source by one unit cell along the waveguide altered the fluxes by about $0.7 \%$. All relevant calculations were repeated with $5 a$ less absorber depth on the $\pm x$ facets to estimate the accuracy of the results, and a change of the measured fluxes of a few $\%$ was observed.

The simulated efficiency $\eta_{\mathrm{c}}$ and reflectivity $R_{\mathrm{c}}$ is shown in Fig. 19(a) as a function of energy for the fundamental WG mode. Note that the coupler efficiency of about $40 \%$ is referring to a single-sided emission, and that double-sided efficiencies are twice as high considering the reflection symmetry in $z$.

We note that we find a reflectivity $R_{\mathrm{c}}$ of $10 \%-20 \%$ for the odd mode in the relevant QD energy range (see Fig. 3), while the measured circularity provides an upper limit (see Appendix E) of about 5\%. There are two main aspects contributing to this difference: (i) the singly reflected light 


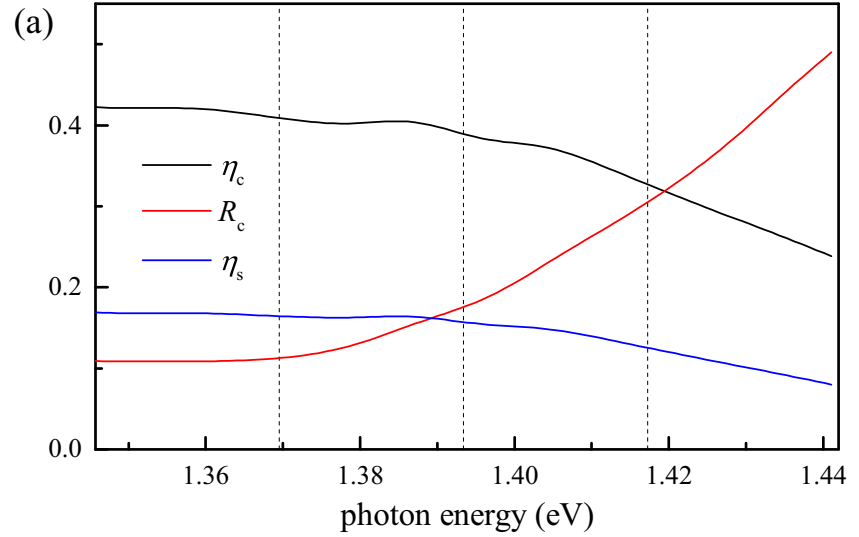

(b)
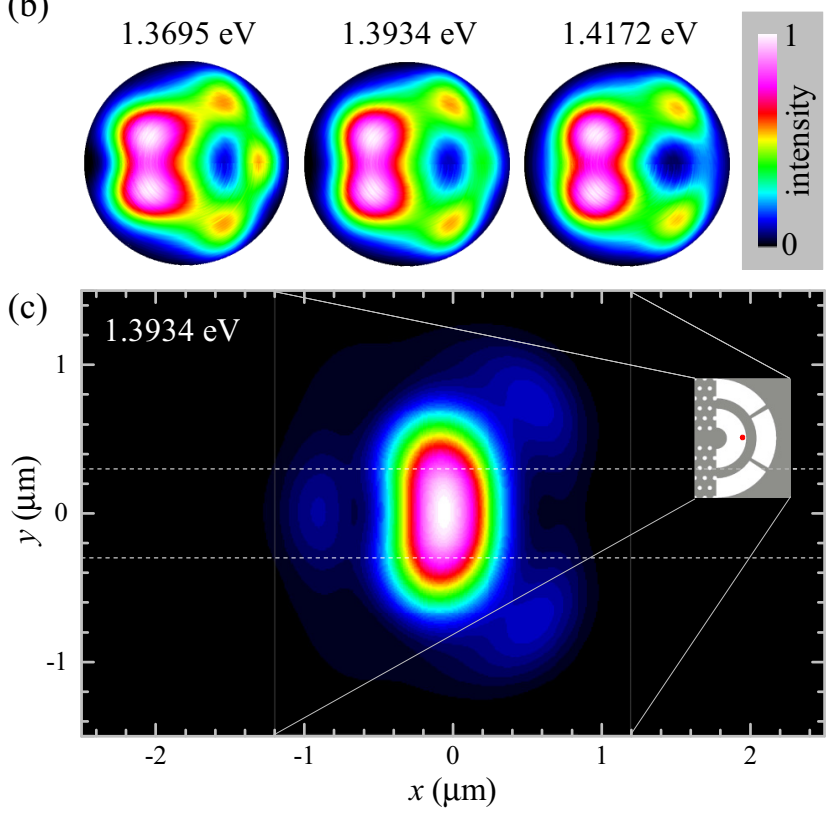

FIG. 19. (a) Simulated efficiency $\eta_{\mathrm{c}}$ (black line) and reflectivity $R_{\mathrm{c}}$ (red line) of the grating coupler for the odd (fundamental) WG mode as a function of energy. The blue line indicates the coupler efficiency into the spectrometer $\eta_{\mathrm{s}}$, taking into account the MO NA and the spectrometer slit. (b) Normalized far-field intensity of the coupler for three selected energies. The radius of the plots is $k_{0}$. (c) Simulated near-field image at $1.3934 \mathrm{eV}$ for a $\mathrm{MO}$ of $0.85 \mathrm{NA}$, imaged with a magnification of 31.3 onto the spectrometer slit. The scale given refers to the size at the sample. The size of the spectrometer input slit is shown by the dashed lines. Inset: sketch of the coupler; the red dot indicates $x=y=0 \mu \mathrm{m}$.

propagates up to $2 L$ further. Using the typical propagation losses of $0.02 / \mu \mathrm{m}$ (see Fig. 7) over a distance of $L$ we find a transmission factor of 0.6 . (ii) $P_{\text {ic }}$ is measured close to the coupler, and thus $R_{\mathrm{c}}$ contains contributions from nonguided modes. For the even mode, the light reflected is furthermore distributed between the two branches inside and outside of the light cone, with the former having strong propagation losses. Both aspects reduce the reflectivity relevant for the measured circularity.

In order to simulate the efficiency of coupling into the spectrometer, the far field of the couplers was extracted from the simulation. The MEEP near-field to far-field transform is used to calculate the full six-component electromagnetic field vector at points homogeneously sampling a hemisphere of radius $R=10^{6} a$, much larger than any simulation feature. The resulting far-field intensity distribution in $k_{x}, k_{y}$ is indicated in Fig. 19(b) for three frequencies. The far field was then limited to the objective collection range, and transformed back into the near field. In detail, for each far-field point, having $x, y, z$ coordinates on the hemisphere, we calculate the in-plane wave vector $\mathbf{k}=k_{0}[x, y] / R$. We then rotate the six-component electromagnetic field at that point from the radial to the $z$ propagation direction, resulting in $E_{z}=H_{z}=0$, and simulating the transformation of the emitted field by the aplanatic objective into its back focal plane. The imaging of the coupler onto the spectrometer slit is then calculated by multiplying the field at each point with a $2 \mathrm{D}$ plane wave $\exp (i \mathbf{k r})$, and summing the vector-field plane waves from every far-field point within the MO NA $\left(|\mathbf{k}|<0.85 k_{0}\right)$. This procedure provides the near field in a small-angle approximation, valid for the small NA of about 0.03 of the image at the spectrometer slit, which is magnified by a factor of 31.3. This near field [see Fig. 19(c)] is then transmitted through the spectrometer slit (dashed lines), providing a near-field collection efficiency. The coupler efficiency, and the near-field collection efficiency, are multiplied to produce the spectrometer efficiency $\eta_{\mathrm{s}}$ [see Fig. 19(a)], which averages to $14.4 \%$ in the simulated range. This is the efficiency with which photons emitted by a QD into the PCWG mode will enter the spectrometer, neglecting propagation loss in the WG. Losses occurring for all detected light, such as reflection loss of the optics, and detector quantum efficiency, are not considered here as they are not influencing the measured beta factor $\beta_{\mathrm{m}}$. These are estimated to provide an additional factor of around $30 \%$ in the setup used, resulting in an estimated overall detection efficiency of QD emission around $4 \%$.

The analysis above used the sum of the intensities of the two polarization components of the propagating field. To take into account the polarization dependence of the detection efficiency, due to the diffraction grating of the spectrometer, we separated the intensity transmitted through the spectrometer slit into $x$ - and $y$-polarized components, and find $18 \%$ of the power in $x$ and $82 \%$ in $y$ polarization, at $E=1.3934 \mathrm{eV}$.

We performed an equivalent analysis for the even mode, as shown in Fig. 20. This mode shows two minima and one maximum within the PC band gap. Close to these extrema, the mode group velocity tends to zero, thus producing a slow light regime, which gives rise to difficulties in the numerical treatment, leading to unreliable results above $1.42 \mathrm{eV}$ and below $1.385 \mathrm{eV}$ in our simulations. In the remaining range, the calculated coupler efficiency is slightly lower and the reflectivity is significantly higher than for the odd mode. The far-field pattern [see Fig. 20(b)] is more structured, and the near field [see Fig. 20(c)] is wider than for the odd mode. Overall, this results in a collection efficiency $\eta_{\mathrm{s}}$ of the higherorder mode of $10 \%$ at $E=1.403 \mathrm{eV}$, of which $73 \%$ is $x$ polarized.

To determine the corrected $\tilde{\beta}_{\mathrm{s}}$ for Eq. (5), we have used a collection efficiency of $14 \%$ for the fundamental mode, calculated as the average over the simulated range, and $10 \%$ for the higher-order mode. 
(a)

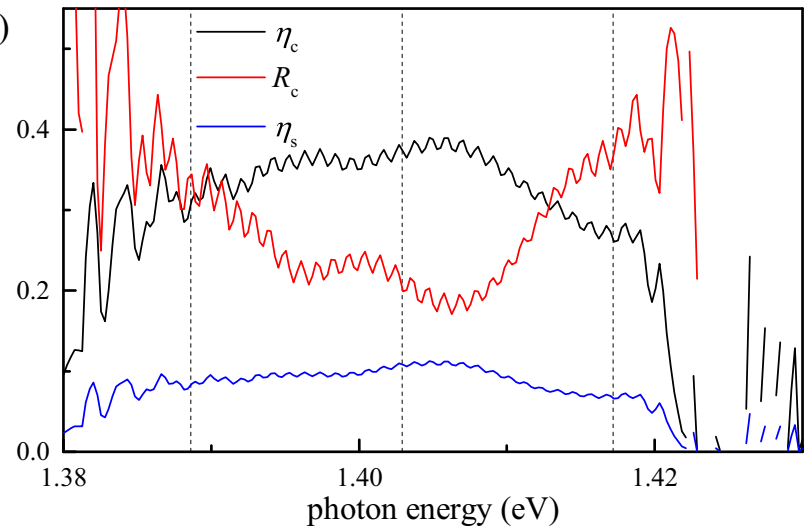

(b)
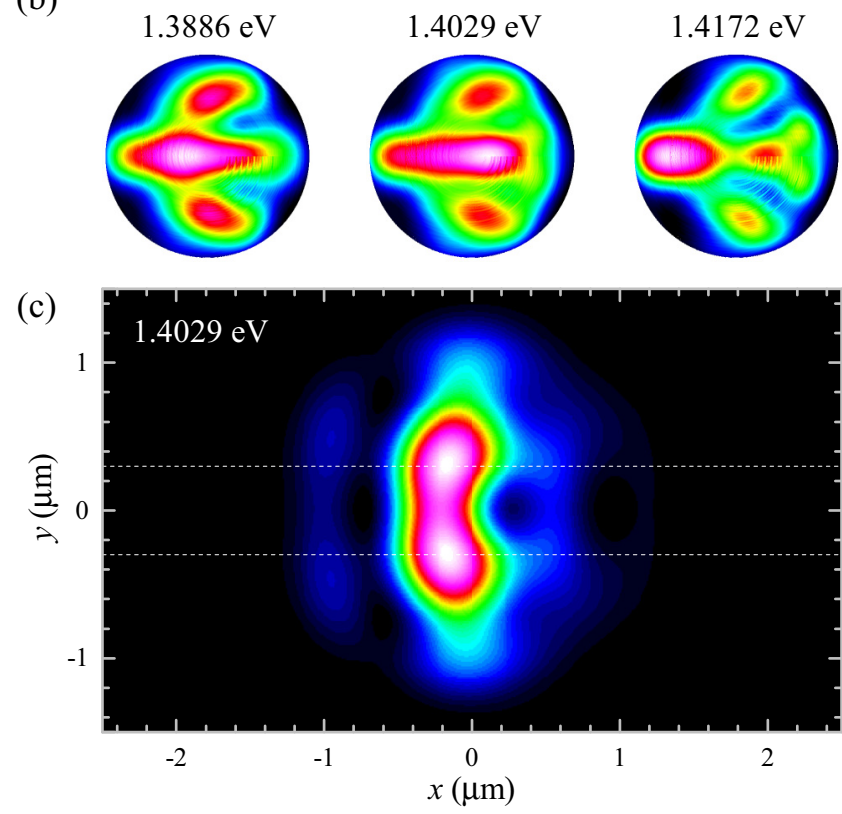

FIG. 20. As Fig. 19, but for the even (higher-order) WG mode.

\section{Beta factor and directionality}

The beta factor and directionality were calculated using further FDTD simulations. We use a simulation volume of size $39 a$ in $x, 18 a$ in $y$, and $5 a$ in $z$ direction, as sketched in Fig. 21. A circular dipole source, $\mathbf{d}=[i, 1]$, is placed in the central unit cell at $z=0$, at a given position in $x, y$. The source has the same time dependence as used for the coupler simulation in Appendix G 3. The beta factor and directionality of the source is calculated from the power the source radiates into the different channels. To obtain accurate results, we have to suppress reflections from the boundaries of the simulation volume. Similar to Appendix G3, we use adiabatic absorbers of $15 a$ thickness covering the $\pm x$ boundaries of the simulation [43], leaving the central nine unit cells unperturbed. In the $y$ direction, the photonic band gap results in a strong reduction of the field, and the boundaries are in the region of unstructured slab, so that absorbers of $2 a$ thickness were found to suffice. In the $z$ direction, extending in free space, PMLs of thickness $a$ were used. In the $\pm x$ directions the absorption profile is quartic while in the others it is quadratic. The mirror symmetry at $z=0$ is exploited in the calculation.
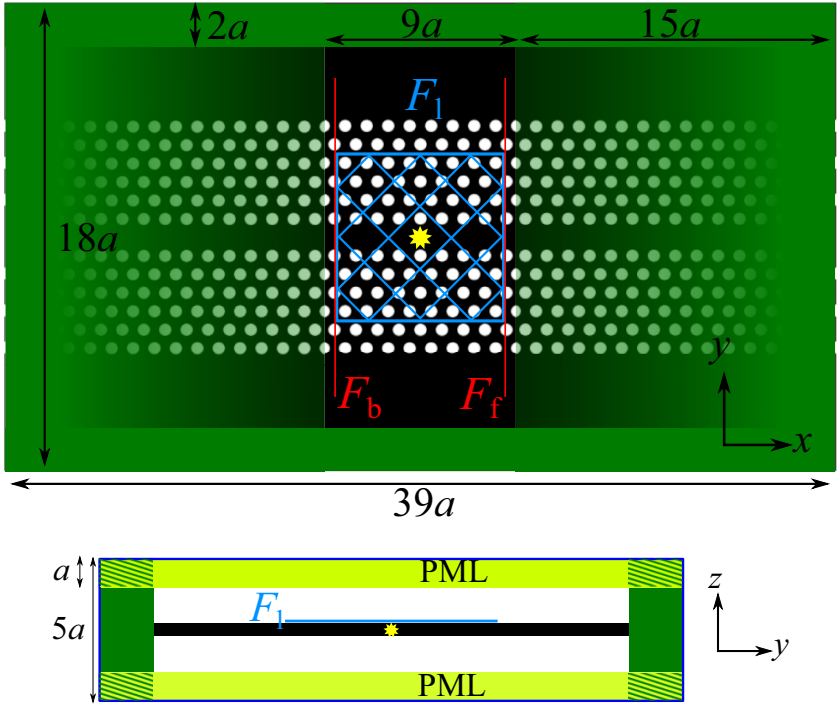

FIG. 21. Sketch of the simulation volume used to calculate the beta factor and directionality. Colors and symbols have the same meanings as in Fig. 18.

Flux planes in the WG forward and backward directions record the frequency-resolved transmitted powers $F_{\mathrm{f}}$ and $F_{\mathrm{b}}$, respectively. They are placed $4 a$ away from the source in $x$, and are $15 a$ wide in $y$, covering the entire photonic cladding but not the $\pm y$ absorbers. In $z$ they extend $0.05 a$ beyond either side of the slab, to contain the mode in the WG and most of its evanescent tail, while limiting the overlap with nonguided light. A third flux plane records the power radiated out of the slab $\left(F_{1}\right)$ in the $+z$ direction, which is doubled to account for the symmetry. These three quantities determine the beta factor and the directionality by

$$
\beta_{\mathrm{s}}=\frac{F_{\mathrm{f}}+F_{\mathrm{b}}}{F_{\mathrm{f}}+F_{\mathrm{b}}+F_{\mathrm{l}}}, \quad D_{\mathrm{s}}=\frac{F_{\mathrm{f}}-F_{\mathrm{b}}}{F_{\mathrm{f}}+F_{\mathrm{b}}} .
$$

Note that $\beta_{\mathrm{s}}$ refers to the total fraction of the QD emission into all guided modes and at each frequency we consider there can be up to six such modes.

In a single-mode waveguide hosting a circular dipole source, $D$ at each location and frequency is equal to the third Stokes parameter $S_{3}$ of the mode field. In multimode waveguides, $D$ is given by an average of the mode $S_{3}$ 's weighted by their densities of states [44]. For comparison with experiment, $D$ can be converted into a circularity value by

$$
C=\frac{1}{2} \log \left(\frac{1+D}{1-D}\right) \text {. }
$$

In each simulation these quantities are extracted as a function of frequency for a given source location. To probe the spatial dependence of the beta factor and circularities, simulations with the source dipole at different locations were performed. As the dipole source is moved in the $x$ direction, it is moved closer to one waveguide flux plane and further from the other. For modes with propagation loss this will impact the measured directionality as the light travels further in one direction than the other. To compensate for this, all calculations were run twice, once with the dipole at $+x$ and again at $-x$. The lattice symmetry ensures that the directionality 

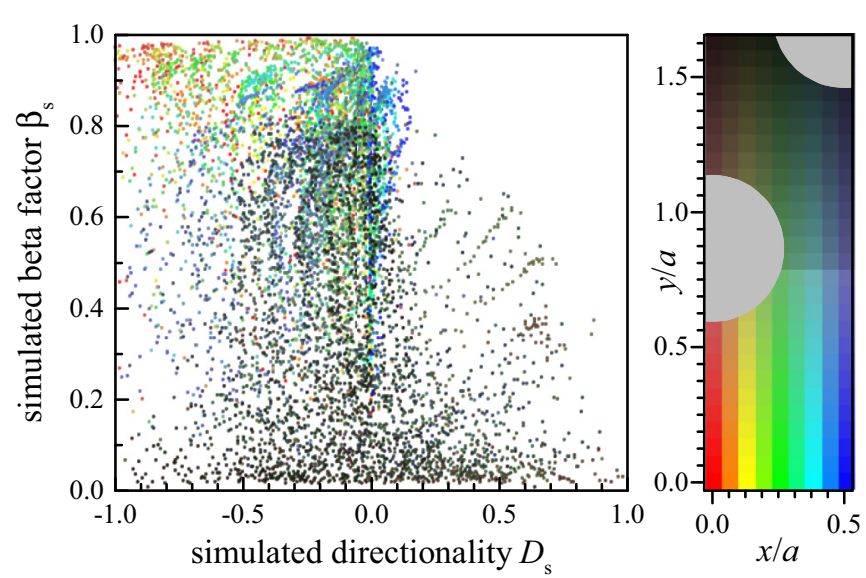

FIG. 22. Simulated beta factor $\beta_{\mathrm{s}}$ versus simulated directionality $D_{\mathrm{s}}$ for 100 equidistant energies from 1.3457 to $1.4411 \mathrm{eV}$. The color of the data points corresponds to their locations as shown in the right panel. Positions inside the holes (light gray) are not considered.

must be identical at these two locations. Averaging the powers of the two simulations accounts for the effect of being closer to one flux plane or the other, removing the impact of losses on the directionality, as long as they are small over a unit cell. The effect of the losses on the beta factor remains, due to the propagation loss between the source location and the forward/backward waveguide flux planes. We can see in Fig. 14 that the propagation loss over this distance of about $1 \mu \mathrm{m}$ is negligible $(<3 \%)$ for most of the modes we consider. The only exception is the lossy branch of the even mode, which experiences significant attenuation over this distance. The energy lost by this mode is radiated out of the slab and collected by the flux plane measuring the free-space emission. The mode is so lossy that it is effectively part of the free-space emission, and this is the case also in the measurements.

The beta factors $\beta_{\mathrm{s}}$ inferred from these calculations are plotted in Fig. 22 versus the directionality. Each data point corresponds to a location in the WG unit cell and an emission energy. The locations are color coded according to the map shown. The hue varies along the $x$ axis and the saturation along $y$. Note that the breaking of symmetry in $D_{\mathrm{s}}$ originates from the display of only half the unit cell, with the other half being mirror symmetric, mapping $x$ to $-x$ and $D_{\mathrm{s}}$ to $-D_{\mathrm{s}}$.

To assess the sensitivity of these results on the choice of the etch parameter $d$, simulations were performed at five random locations with $d=7 \mathrm{~nm}$ instead of $8 \mathrm{~nm}$. After accounting for an overall frequency shift of $0.0014(c / a)(6.7 \mathrm{meV})$, the mean absolute change of $\beta$ was 0.024 , and the change in directionality $D$ was 0.014 . These small changes reflect the fact that the main effect of altering the etch parameter is a shift in frequency while hardly changing the mode profiles.

As discussed in Appendix G3, these simulations will be inaccurate close to the extrema of the even mode, and these energies are not shown in Fig. 22.

\section{Free-space emission}

The far-field intensity distributions in $\mathbf{k}$ space of the freespace emission of circular dipoles at various locations in the PCWG unit cell, taken from the simulations discussed in

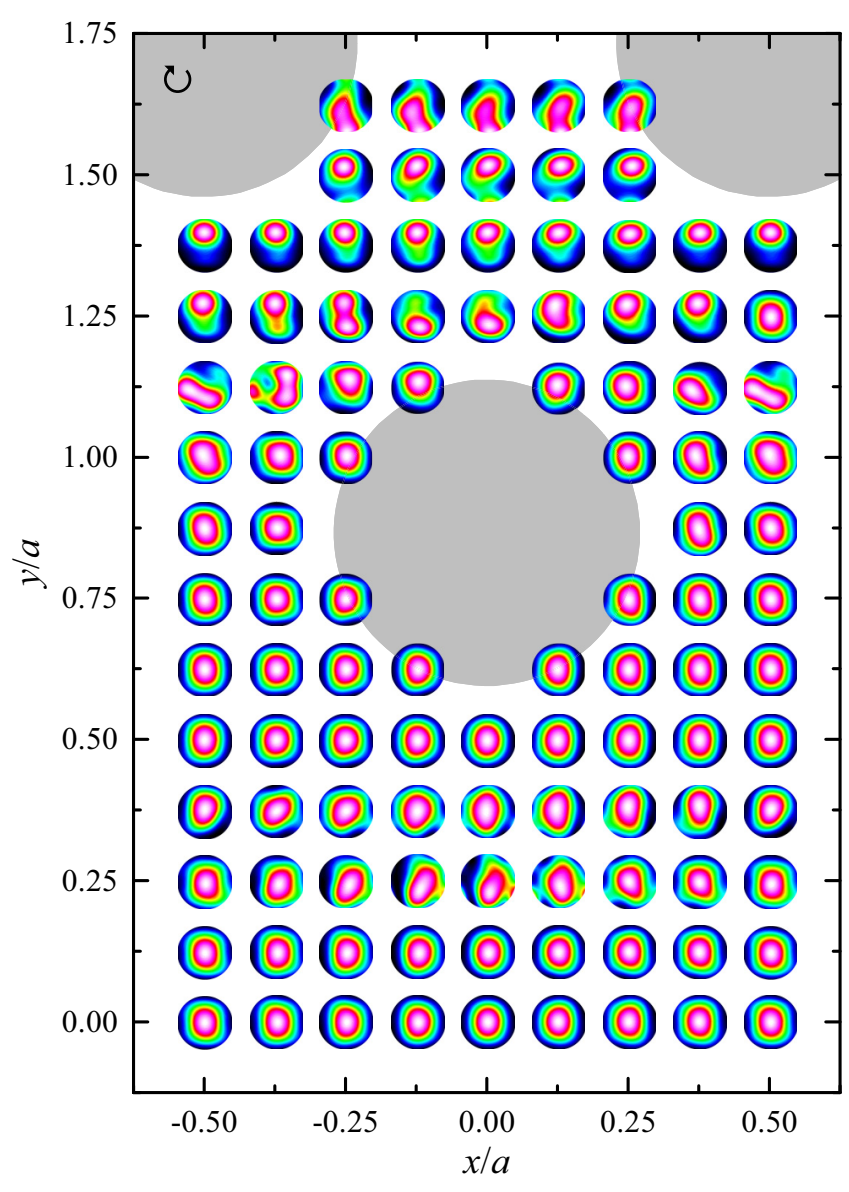

FIG. 23. Far-field profiles of the free-space emission of a righthanded $(\circlearrowright)$ circularly polarized dipole source at $z=0$ with emission energy $E=1.3934 \mathrm{eV}$, as a function of its position $x, y$ within the PCWG unit cell. The circular plots are in $\mathbf{k}$ space having radius of $k_{0}$ using a color scale as shown in Fig. 19.

Appendix G 4, are shown in Fig. 23 for $E=1.3934 \mathrm{eV}$. They show a significant variation with source location. Note that the missing mirror symmetry about $x=0$ arises from the circularly polarized dipole excitation which lacks this symmetry. Using these profiles, we determine the corresponding freespace collection efficiency into the spectrometer, as shown in Fig. 24(b), considering the objective NA and the spectrometer input slit as described in Appendix G3. We can observe that the regions of low values correspond to highly asymmetric far-field profiles in Fig. 23. The average efficiency is about $29 \%$, and varying from $18 \%$ to $33 \%$ with the location of the QD. We can consider these collection efficiencies to improve the accuracy of the beta factors determined in the experiment. We note that free-space emission is collected with up to a factor of 2 higher efficiency than the WG mode emission, suggesting that the real beta factors are up to a factor of 2 closer to unity than the measured beta factor reported in the main text.

To investigate the validity of the assumption of free-space emission strength independent of position, used in previous works reporting on the beta factor, we show in Fig. 24(a) the free-space emission power as function of position, relative to bulk GaAs, for eight energies covering the simulation range. We observe variations over two orders of magnitude, from 


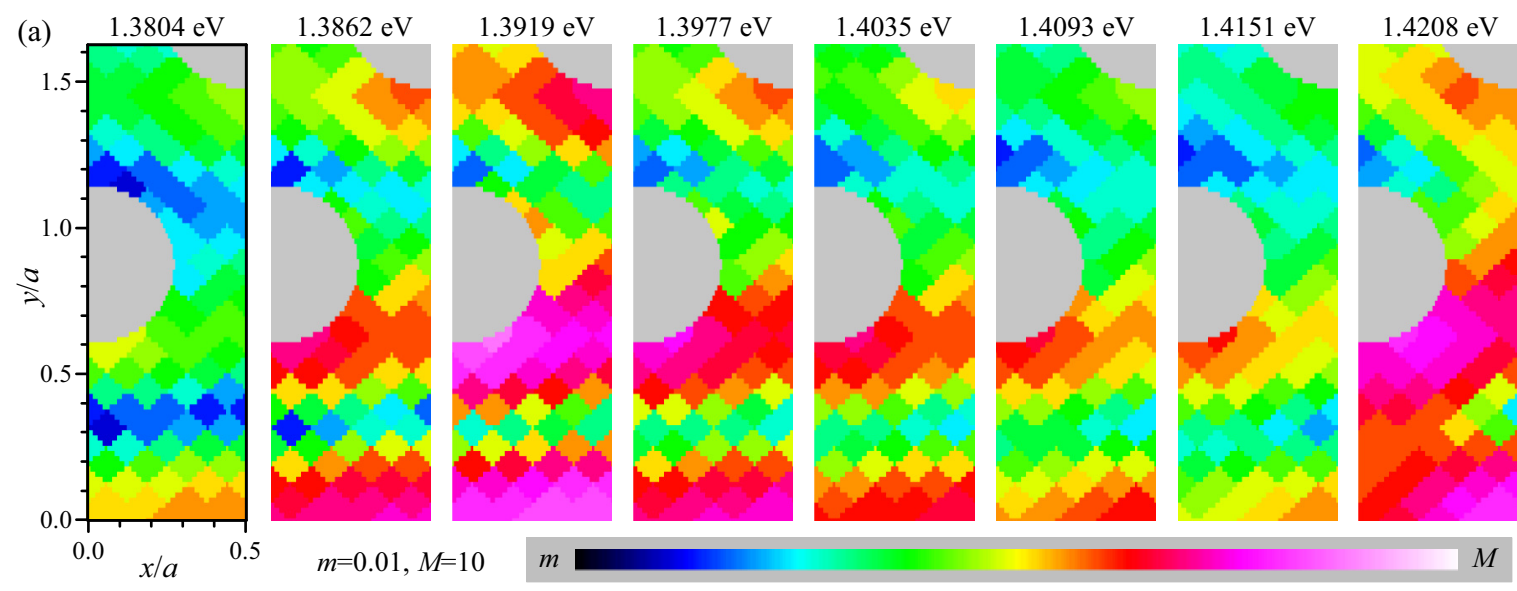

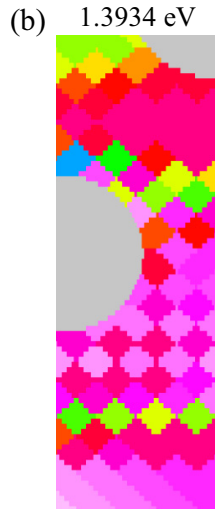

$m=0, M=0.35$

FIG. 24. (a) Emission power for a circular dipole source into nonguided modes, relative to the emission power in bulk GaAs, as a function of source position, for different emission energies as given. Logarithmic color scale from $m$ to $M$ as given. (b) Collection efficiency of the

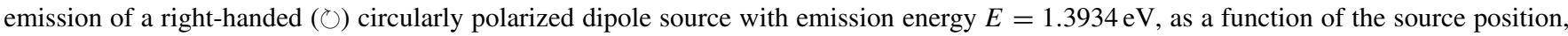
including the objective NA and the input slit of the spectrometer. Linear color scale from $m$ to $M$ as given.

0.05 to 5 , implying that the assumption is not justified. This points toward large systematic errors in the beta factors retrieved in previous works [16-21]. Large spatial variations in the free-space emission are also indicated by the simulations in [24], which also predicts horizontal bands of reduced freespace coupling as seen in Fig. 24.

For $E=1.4208 \mathrm{eV}$, we see large increases in the loss rate. This is attributed to the branch of the even mode in the light cone, as the energy is close to the frequency maximum in the even mode, where its group velocity tends to zero. For a low group velocity the emission is enhanced by the slow light, and the propagation length is reduced, resulting in an emission into free space close to the emitter. Indeed, the pattern resembles the electric field intensity pattern of the even (higher order) seen in Fig. 15.

\section{Mode separation}

The FDTD calculations provide the beta factor and directionality. The values observed in the experiment will be slightly modified from these values by the different collection efficiencies of coupler and free-space emission. As the collection efficiencies of the even and odd modes are different, adjusting for these efficiencies requires separation of the power between the two modes. To achieve this separation, the electrical field profiles $\mathbf{E}(\mathbf{r}, \omega)$ ) and group indices $n_{\mathrm{g}}(\omega)$ extracted from the loss FDTD simulations (see Appendix G 2) are used. The group indices are calculated using the spatially integrated Poynting vector [47]. These are used to calculate the density of states (DOS) into the forward and backward directions at each spatial location using the Purcell factor expressions presented in [1], adapted to separate the intensity in a given direction. As we are only concerned with ratios between modes at a common frequency, these expressions can be simplified to

$$
\begin{gathered}
\operatorname{DOS}_{\text {forward }} \propto|\mathbf{E}(\mathbf{r}, \omega)|^{2} n_{g}(\omega)\left(S_{3}+1\right) / 2, \\
\operatorname{DOS}_{\text {backward }} \propto|\mathbf{E}(\mathbf{r}, \omega)|^{2} n_{g}(\omega)\left(1-S_{3}\right) / 2 .
\end{gathered}
$$

Using the fields associated with each mode, respectively, allows us to calculate the fraction of the power emitted into the forward even mode, for a given location and frequency. We neglect the branch of the even mode in the light cone, as the power in it is lost while propagating to the coupler. Using the resulting fractions, the collection efficiencies of the couplers can be corrected while accounting for the different efficiencies for the two modes.

\section{APPENDIX H: CORRECTION OF SIMULATIONS}

In this Appendix we describe how the raw data presented in Appendix G 4 have been corrected in order to be comparable to the experimental data. The forward $f$ and the backward $b$ propagating fluxes are first separated into the fundamental and higher-order mode projections as calculated in Appendix G6. The fluxes of each mode are then multiplied by the corresponding collection efficiencies due to the couplers, the NA of the MO, and the input slit of the imaging spectrometer. We recall that the collection efficiencies are averaged over the spectral range covered by the simulations. The resulting fluxes from each mode are then separated into transverse and longitudinal polarization components using the analysis presented in Appendix G3. Finally, we include the polarizationdependent efficiency $T$ and $L$ of the spectrometer grating for transverse and longitudinal polarizations, respectively. The polarization resolved fluxes are multiplied by the coefficients $T$ and $L$, and the resulting fluxes are then summed up, separately for each mode, to obtain the final corrected fluxes. $T$ and $L$ are determined by measuring the grating polarization efficiency ratio $R=T / L$, with the normalization $T+L=2$, such that for polarization-independent detection we have $T=$ $L=1$.

For the free-space emission we first multiply the out-ofplane flux by the position-dependent collection efficiency, as described in Appendix G5. In this case, the collection efficiency is calculated at $E=1.3934 \mathrm{eV}$, which is approximately the central energy of the measured QDs. We calculated the energy dependence at one location and we found it to be negligible. In order to include the polarization efficiency of the grating spectrometer, we consider the free-space emission to be circularly polarized. 
[1] V. S. C. Manga Rao and S. Hughes, Phys. Rev. B 75, 205437 (2007).

[2] G. Lecamp, P. Lalanne, and J. P. Hugonin, Phys. Rev. Lett. 99, 023902 (2007).

[3] F. J. Rodríguez-Fortuño, G. Marino, P. Ginzburg, D. O'Connor, A. Martínez, G. A. Wurtz, and A. V. Zayats, Science 340, 328 (2013).

[4] C. Junge, D. O'Shea, J. Volz, and A. Rauschenbeutel, Phys. Rev. Lett. 110, 213604 (2013).

[5] R. Mitsch, C. Sayrin, B. Albrecht, P. Schneeweiss, and A. Rauschenbeutel, Nat. Commun. 5, 5713 (2014).

[6] I. Söllner, S. Mahmoodian, S. L. Hansen, L. Midolo, A. Javadi, G. Kiršanske, T. Pregnolato, H. El-Ella, E. H. Lee, J. D. Song, S. Stobbe, and P. Lodahl, Nat. Nanotechnol. 10, 775 (2015).

[7] A. B. Young, A. C. T. Thijssen, D. M. Beggs, P. Androvitsaneas, L. Kuipers, J. G. Rarity, S. Hughes, and R. Oulton, Phys. Rev. Lett. 115, 153901 (2015).

[8] P. Lodahl, S. Mahmoodian, S. Stobbe, A. Rauschenbeutel, P. Schneeweiss, J. Volz, H. Pichler, and P. Zoller, Nature (London) 541, 473 (2017).

[9] R. Coles, D. Price, J. Dixon, B. Royall, E. Clarke, P. Kok, M. Skolnick, A. Fox, and M. Makhonin, Nat. Commun. 7, 11183 (2016).

[10] R. J. Coles, D. M. Price, B. Royall, E. Clarke, M. S. Skolnick, A. M. Fox, and M. N. Makhonin, Phys. Rev. B 95, 121401(R) (2017).

[11] B. Lang, D. M. Beggs, A. B. Young, J. G. Rarity, and R. Oulton, Phys. Rev. A 92, 063819 (2015).

[12] A. Javadi, D. Ding, M. H. Appel, S. M. M. C. Löbl, I. Söllner, R. Schott, C. Papon, T. Pregnolato, S. Stobbe, L. Midolo, T. Schröder, A. D. Wieck, A. Ludwig, R. J. Warburton, and P. Lodahl, Nat. Nanotechnol. 13, 398 (2018).

[13] J.-H. Kim, S. Aghaeimeibodi, C. J. K. Richardson, R. P. Leavitt, and E. Waks, Nano Lett. 18, 4734 (2018).

[14] P. Lodahl, S. Mahmoodian, and S. Stobbe, Rev. Mod. Phys. 87, 347 (2015).

[15] S. Mahmoodian, P. Lodahl, and A. S. Sørensen, Phys. Rev. Lett. 117, 240501 (2016).

[16] T. Lund-Hansen, S. Stobbe, B. Julsgaard, H. Thyrrestrup, T. Sünner, M. Kamp, A. Forchel, and P. Lodahl, Phys. Rev. Lett. 101, 113903 (2008).

[17] H. Thyrrestrup, L. Sapienza, and P. Lodahl, Appl. Phys. Lett. 96, 231106 (2010).

[18] S. J. Dewhurst, D. Granados, D. J. P. Ellis, A. J. Bennett, R. B. Patel, I. Farrer, D. Anderson, G. A. C. Jones, D. A. Ritchie, and A. J. Shields, Appl. Phys. Lett. 96, 031109 (2010).

[19] A. Laucht, S. Putz, T. Gunthner, N. Hauke, R. Saive, S. Frederick, M. Bichler, M. C. Amann, A. W. Holleitner, M. Kaniber, and J. J. Finley, Phys. Rev. X 2, 011014 (2012).

[20] T. B. Hoang, J. Beetz, L. Midolo, M. Skacel, M. Lermer, M. Kamp, S. Höfling, L. Balet, N. Chauvin, and A. Fiore, Appl. Phys. Lett. 100, 061122 (2012).

[21] M. Arcari, I. Sollner, A. Javadi, S. LindskovHansen, S. Mahmoodian, J. Liu, H. Thyrrestrup, E. H. Lee, J. D. Song, S. Stobbe, and P. Lodahl, Phys. Rev. Lett. 113, 093603 (2014).

[22] A. Kurzmann, A. Ludwig, A. D. Wieck, A. Lorke, and M. Geller, Nano Lett. 16, 3367 (2016).
[23] W. Langbein, P. Borri, U. Woggon, V. Stavarache, D. Reuter, and A. D. Wieck, Phys. Rev. B 70, 033301 (2004).

[24] A. Javadi, S. Mahmoodian, I. Söllner, and P. Lodahl, J. Opt. Soc. Am. B 35, 514 (2018).

[25] B. Rigal, B. Dwir, A. Rudra, I. Kulkova, A. Lyasota, and E. Kapon, Appl. Phys. Lett. 112, 051101 (2018).

[26] P. Tighineanu, C. L. Dreeßen, C. Flindt, P. Lodahl, and A. S. Sørensen, Phys. Rev. Lett. 120, 257401 (2018).

[27] W. Langbein, P. Borri, U. Woggon, V. Stavarache, D. Reuter, and A. D. Wieck, Phys. Rev. B 69, 161301(R) (2004).

[28] M. Bayer, G. Ortner, O. Stern, A. Kuther, A. A. Gorbunov, A. Forchel, P. Hawrylak, S. Fafard, K. Hinzer, T. L. Reinecke, S. N. Walck, J. P. Reithmaier, F. Klopf, and F. Schäfer, Phys. Rev. B 65, 195315 (2002).

[29] S. G. Johnson, S. Fan, P. R. Villeneuve, J. D. Joannopoulos, and L. A. Kolodziejski, Phys. Rev. B 60, 5751 (1999).

[30] A. Faraon, I. Fushman, D. Englund, N. Stoltz, P. Petroff, and J. Vučković, Opt. Express 16, 12154 (2008).

[31] A. A. Sukhorukov, S. Ha, I. V. Shadrivov, D. A. Powell, and Y. S. Kivshar, Opt. Express 17, 3716 (2009).

[32] H. Gersen, T. J. Karle, R. J. P. Engelen, W. Bogaerts, J. P. Korterik, N. F. van Hulst, T. F. Krauss, and L. Kuipers, Phys. Rev. Lett. 94, 073903 (2005).

[33] A. Laucht, T. Günthner, S. Pütz, R. Saive, S. Frédérick, N. Hauke, M. Bichler, M.-C. Amann, A. W. Holleitner, M. Kaniber, and J. J. Finley, J. Appl. Phys. 112, 093520 (2012).

[34] W. Langbein, J. M. Hvam, and R. Zimmermann, Phys. Rev. Lett. 82, 1040 (1999).

[35] W. Langbein and J. M. Hvam, Phys. Rev. Lett. 88, 047401 (2002).

[36] N. L. Thomas, R. Houdré, M. V. Kotlyar, D. O’Brien, and T. F. Krauss, J. Opt. Soc. Am. B 24, 2964 (2007).

[37] S. Gehrsitz, F. K. Reinhart, C. Gourgon, N. Herres, A. Vonlanthen, and H. Sigga, J. Appl. Phys. 87, 7825 (2000).

[38] F. Lukeš, Surf. Sci. 30, 91 (1972).

[39] G. C. DeSalvo, C. A. Bozada, J. L. Ebel, D. C. Look, J. P. Barrette, C. L. A. Cerny, R. W. Dettmer, J. K. Gillespie, C. K. Havasy, T. J. Jenkins, K. Nakano, C. I. Pettiford, T. K. Quach, J. S. Sewell, and G. D. Via, J. Electrochem. Soc. 143, 3652 (1996).

[40] Cardiff University data archive, http://doi.org/10.17035/d.2019. 0067980126.

[41] Y. Benny, Y. Kodriano, E. Poem, D. Gershoni, T. A. Truong, and P. M. Petroff, Phys. Rev. B 86, 085306 (2012).

[42] A. F. Oskooi, D. Roundy, M. Ibanescu, P. Bermel, J. Joannopoulos, and S. G. Johnson, Comput. Phys. Commun. 181, 687 (2010).

[43] A. Oskooi, L. Zhang, Y. Avniel, and S. Johnson, Opt. Express 16, 11376 (2008).

[44] B. Lang, D. M. Beggs, and R. Oulton, Philos. Trans. R. Soc. A 374, 2075 (2016).

[45] Y. Akahane, T. Asano, B.-S. Song, and S. Noda, Opt. Express 13, 1202 (2005).

[46] S. G. Johnson and J. D. Joannopoulos, Opt. Express 8, 173 (2001).

[47] Y. Chen, T. R. Nielsen, N. Gregersen, P. Lodahl, and J. Mørk, Phys. Rev. B 81, 125431 (2010). 